
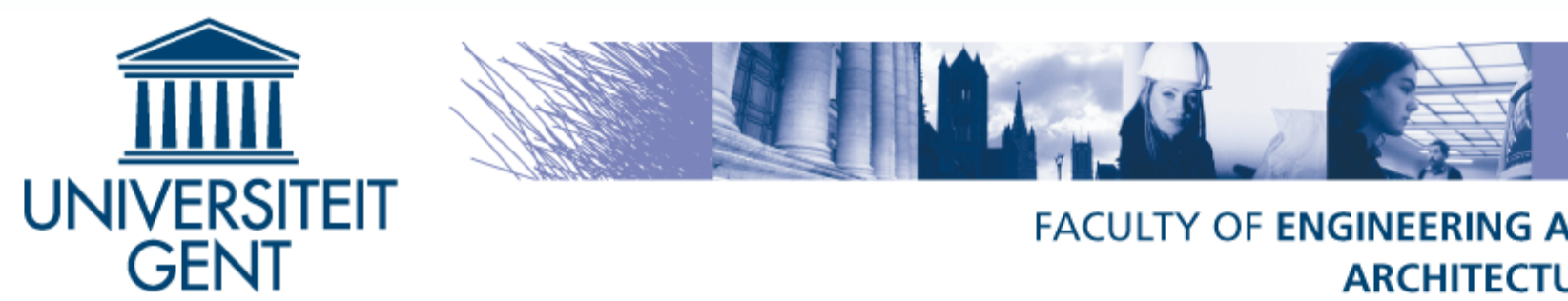

FACULTY OF ENGINEERING AND ARCHITECTURE

\title{
Modeling Fast Biomass Pyrolysis in a Gas/Solid Vortex Reactor
}

Robert W. Ashcraft, Jelena Kovacevic, Geraldine J. Heynderickx, Guy B. Marin

Laboratory for Chemical Technology, Ghent University http://www.Ict.UGent.be 


\section{History and Description}




\section{Vortex Reactor Development Timeline}

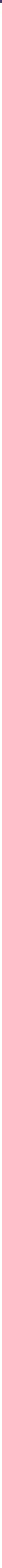




\section{Gas/Solid Vortex Reactor (GSVR)}

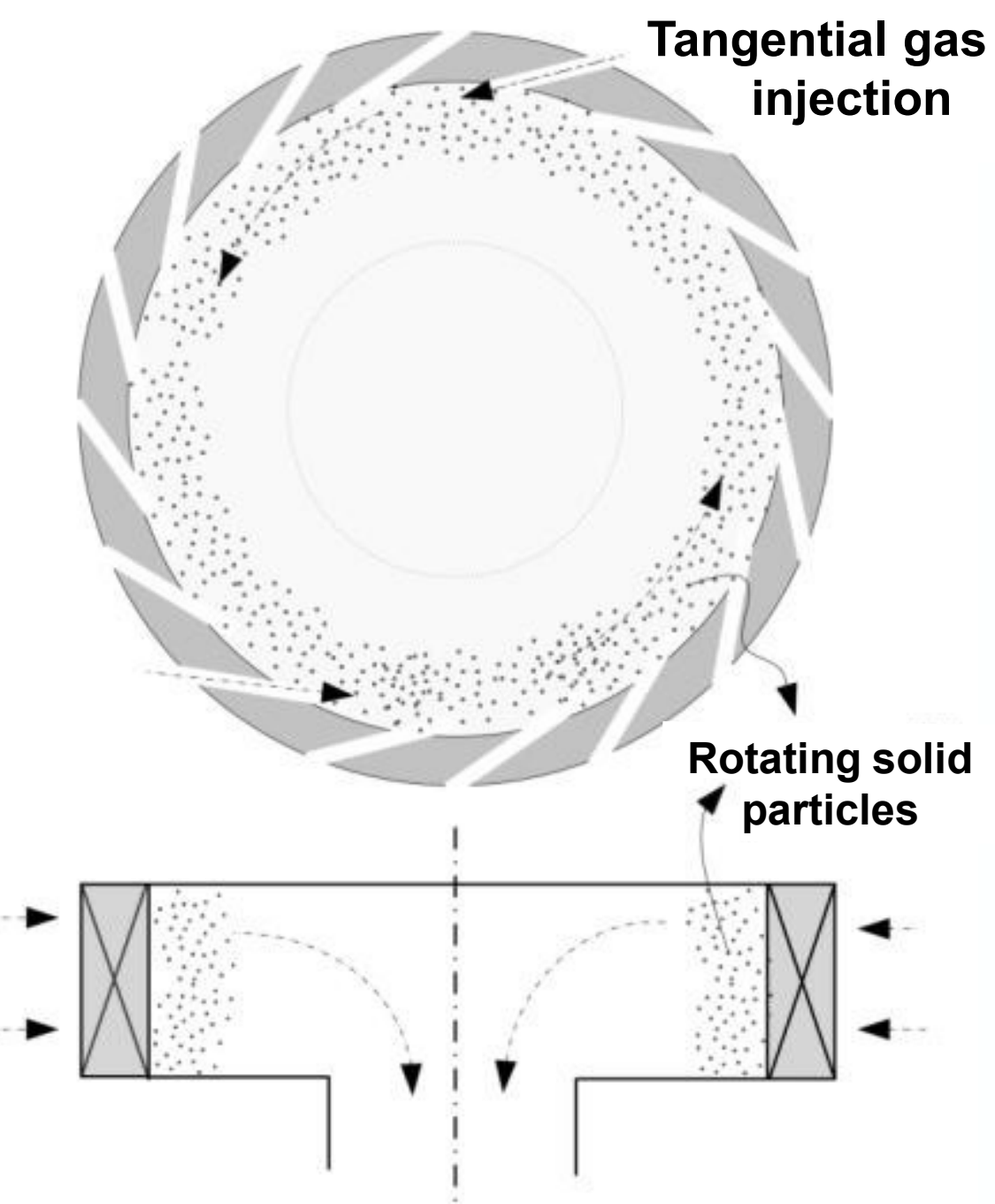

\section{GSVR Characteristics:}

- Gas injection forces bed rotation \& induces fluidization

- Centrifugal forces resist drag

$>$ Dense bed

$>$ High radial slip velocity

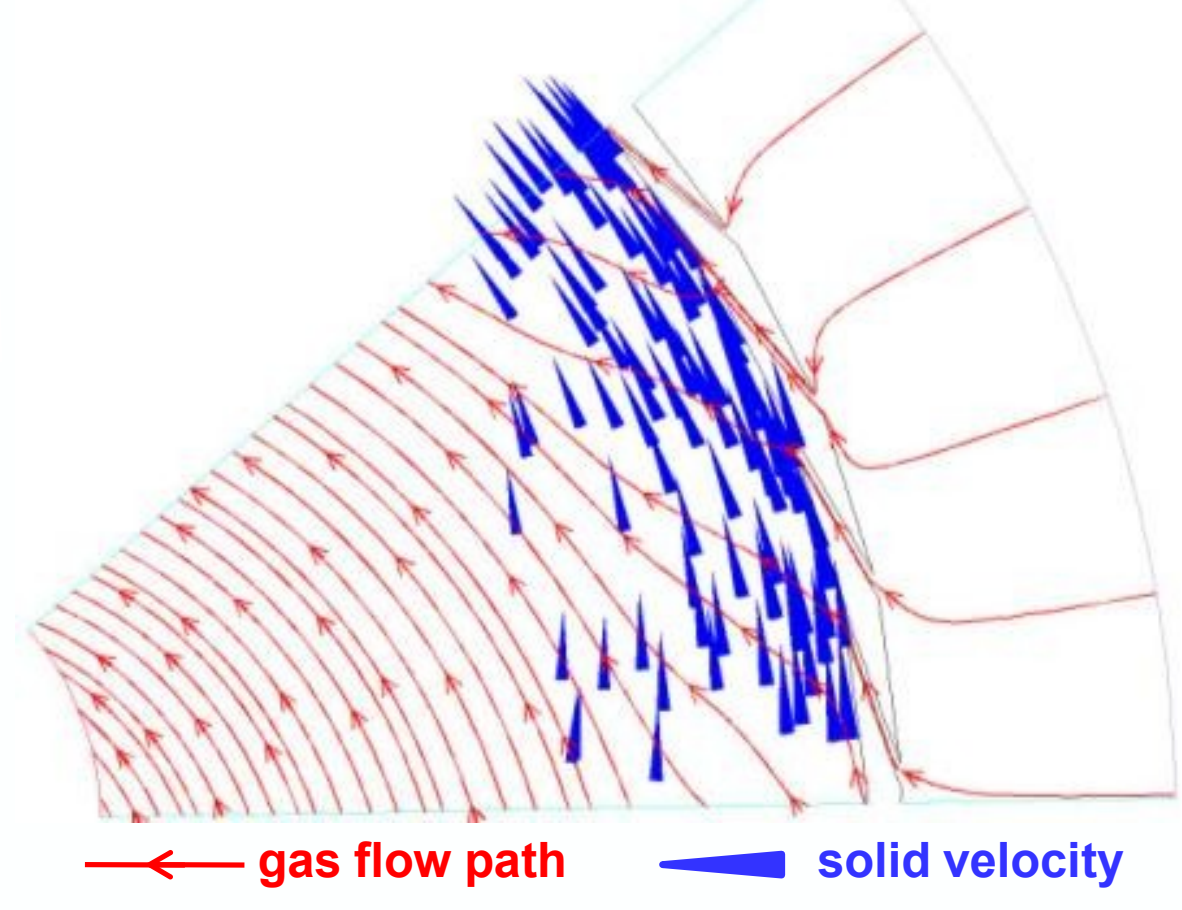




\section{General Vortex Reactors}

\section{Rotating Bed Reactors in a Static Geometry (RBR-SG)}
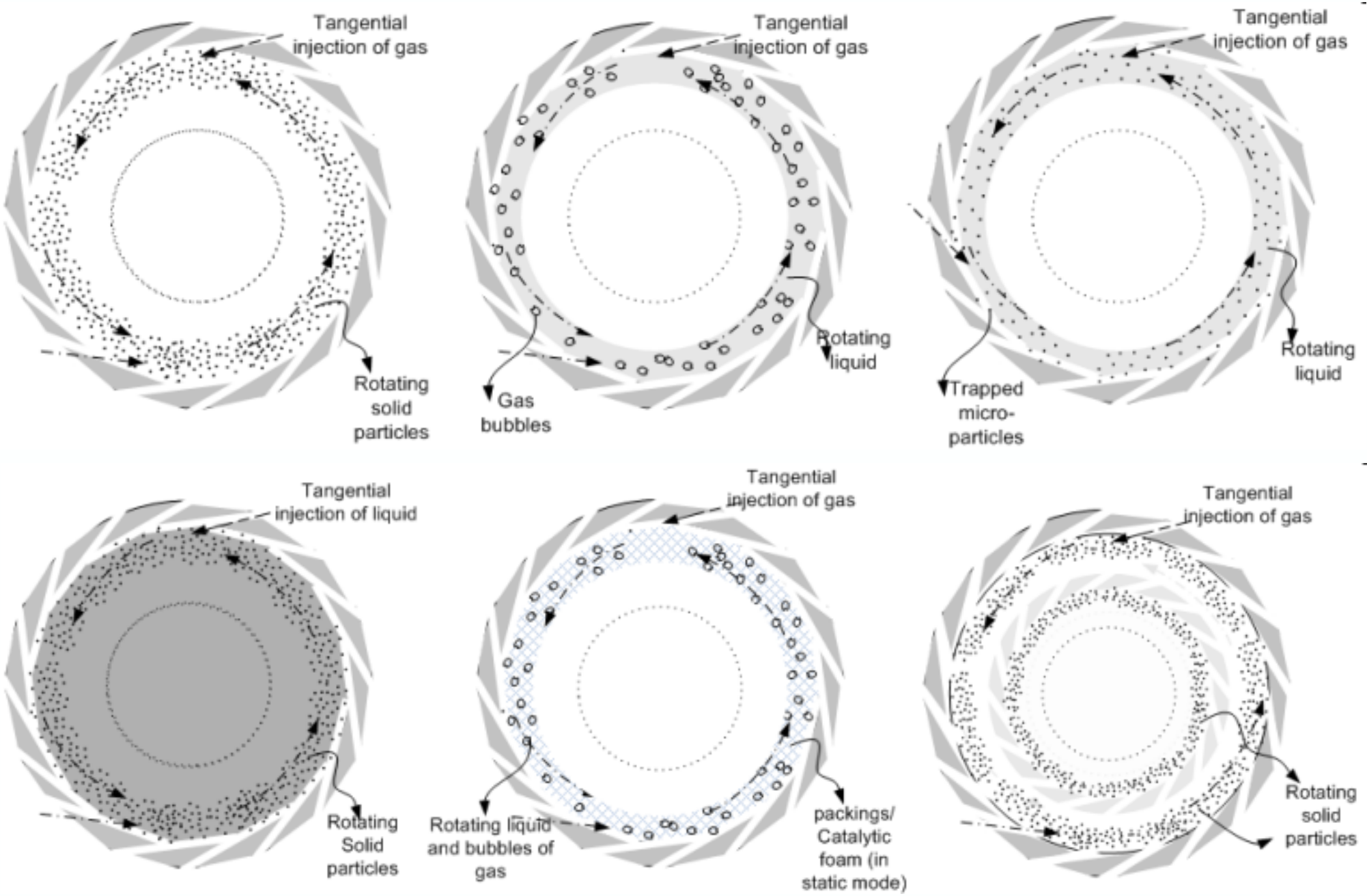

[Anderson et al, 1972], [Kuzmin et al, 2005], [Loftus et al, 1992, Fichman, et al, 2008], [Haldipur P, 1999], [Trachuk A. V., 2009], [Entoleter Inc, 1973] 


\section{Gas/Solid Fluidization Reactors}

\section{Conventional Fluidized Bed ${ }^{1}$}

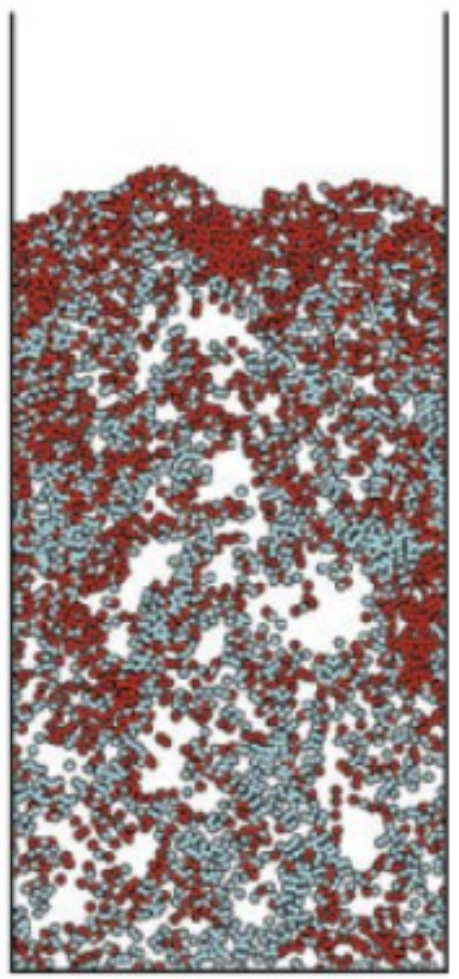

Riser/Circulating Fluidized Bed ${ }^{2}$

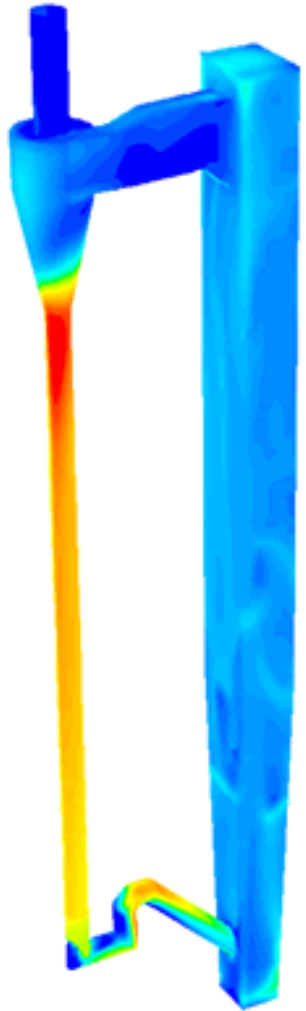

Conventional Rotating Fluidized Bed ${ }^{3}$

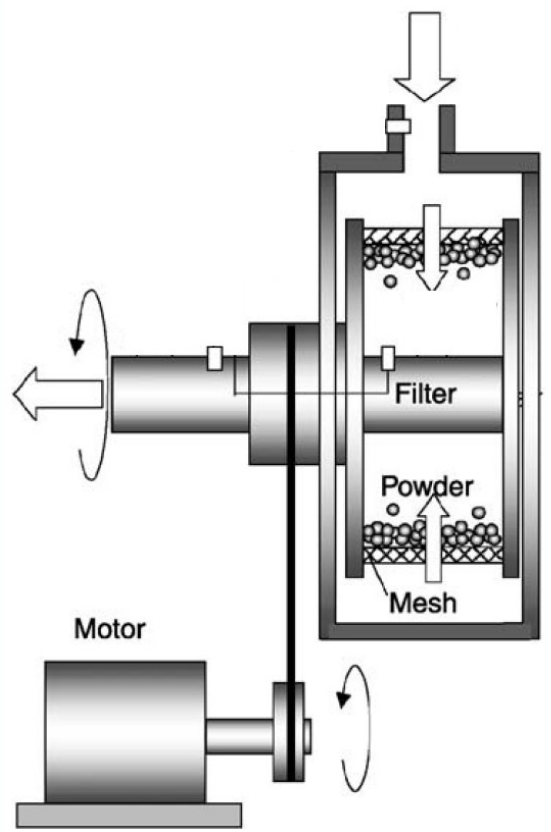

Gas/Solid Vortex Reactor

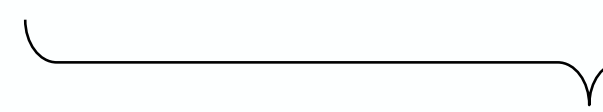

gravitational technologies

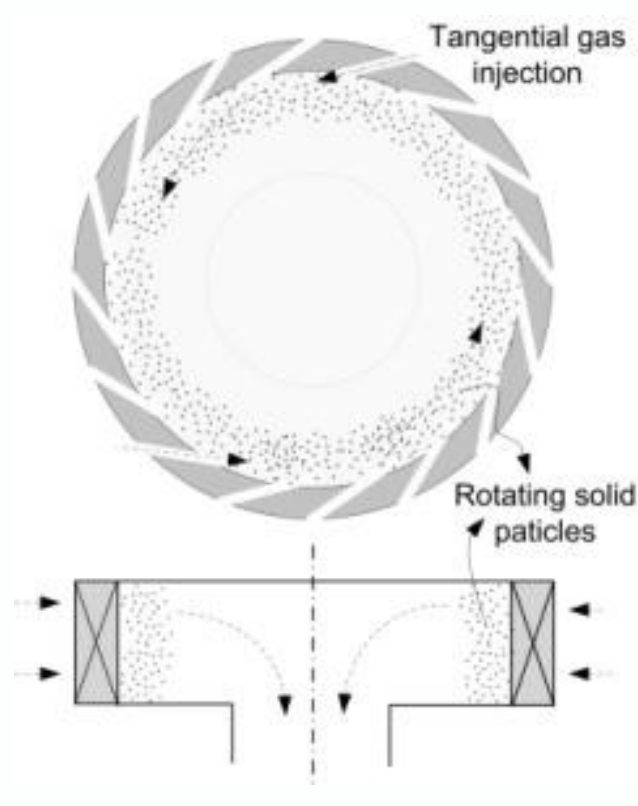

1. van Hoef et al., Ann. Rev. Fluid Mech. 40 (2008) 47-70

2. http://www.fluidcodes.co.uk/fbed.html

3. adapted from Watano et al., Powder Tech.131 (2003) 250-255 


\section{Gas/Solid Fluidization Reactors}

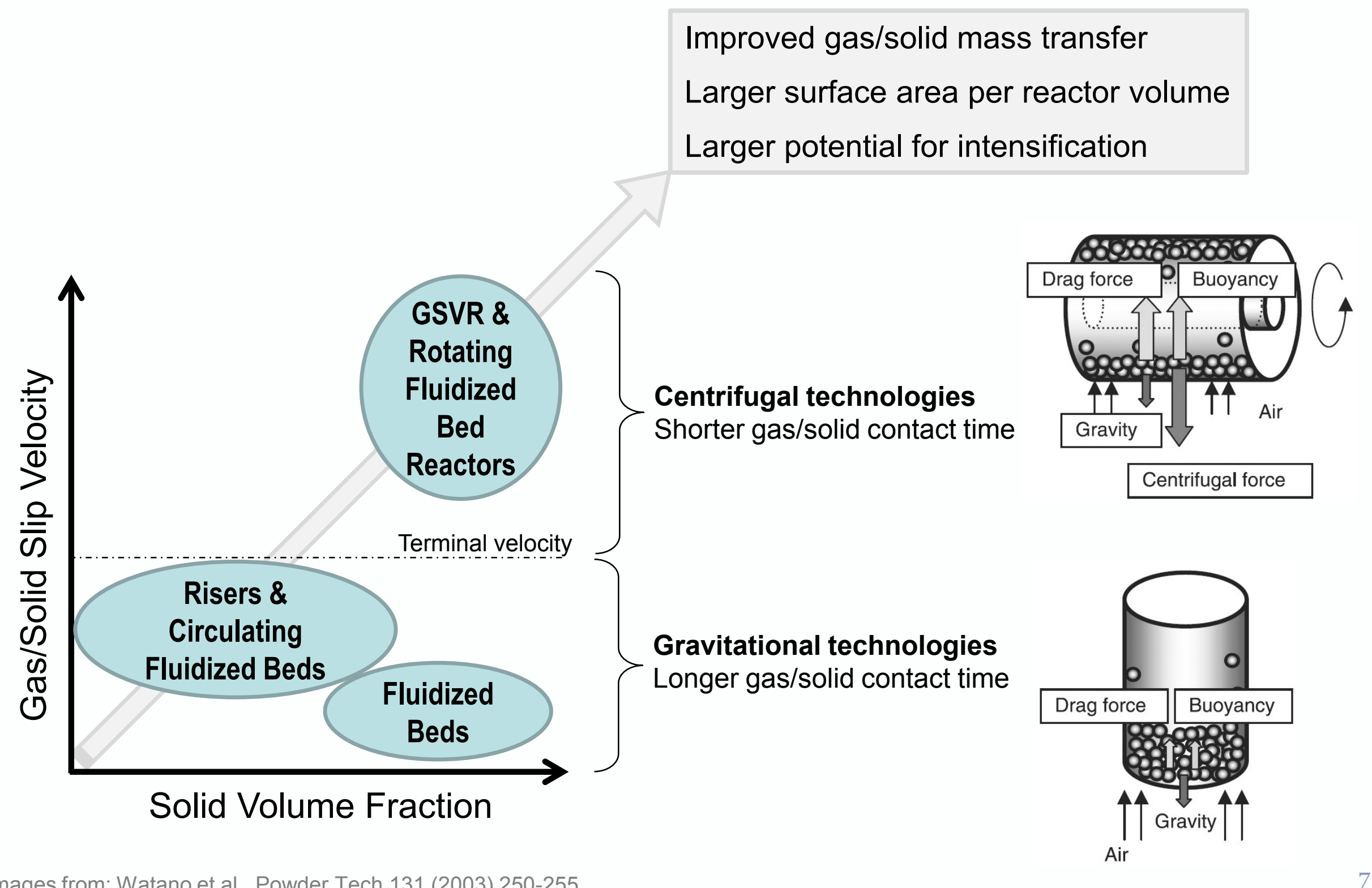




\section{Experimental}

with Jelena Kovacevic 


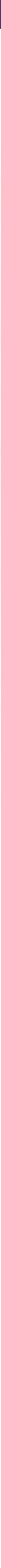




\section{Real-time Video}

$0.9 \mathrm{~mm}$ polyvinylidene fluoride particles $\left(\rho=1800 \mathrm{~kg} / \mathrm{m}^{3}\right)$

$\sim 1 \mathrm{~kg} / \mathrm{s}$ air flow

$\sim 5 \mathrm{~kg}$ bed mass

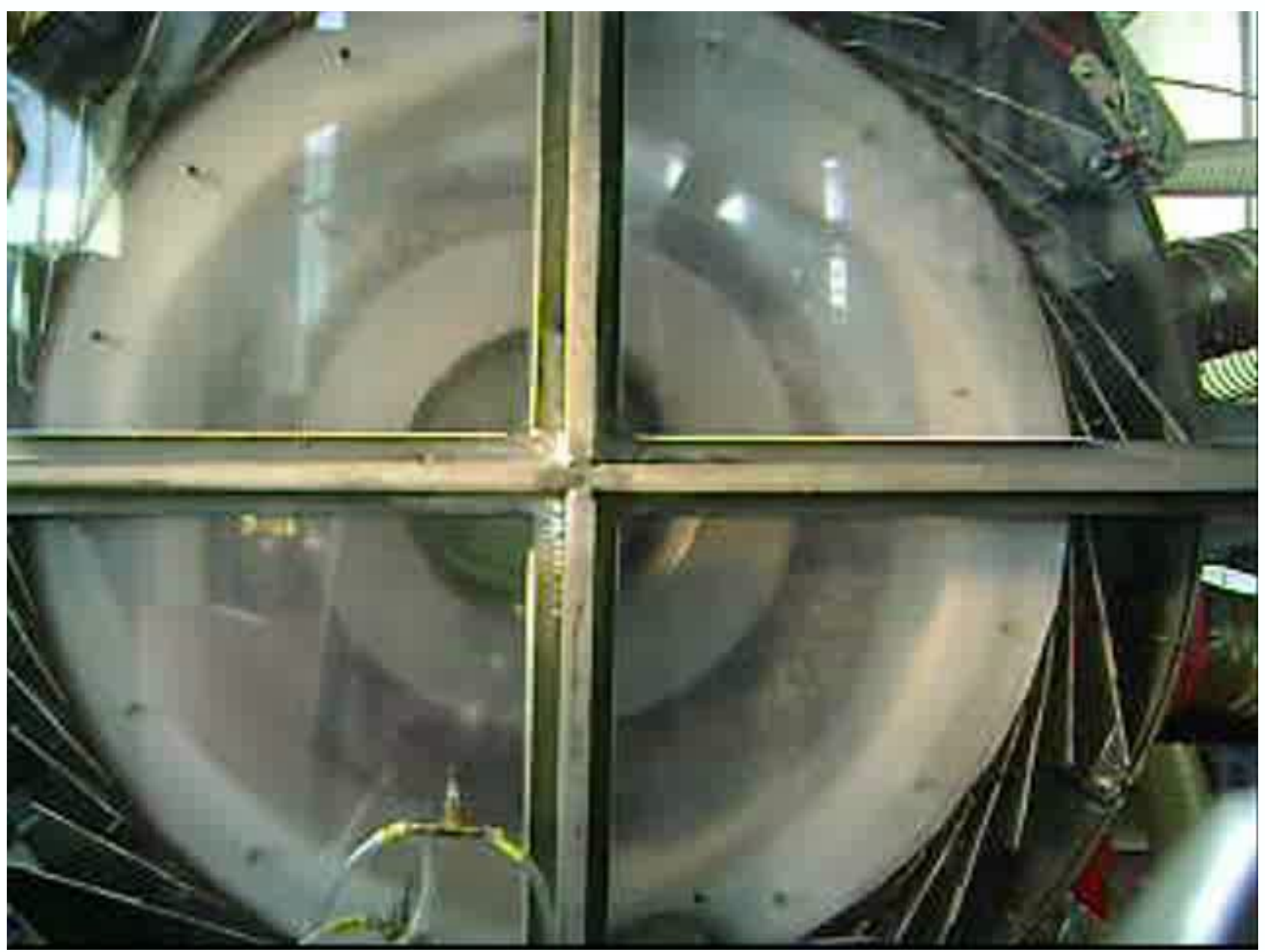




\section{High-Speed Video - Small Particles}

70 micron particles (5000 FPS)

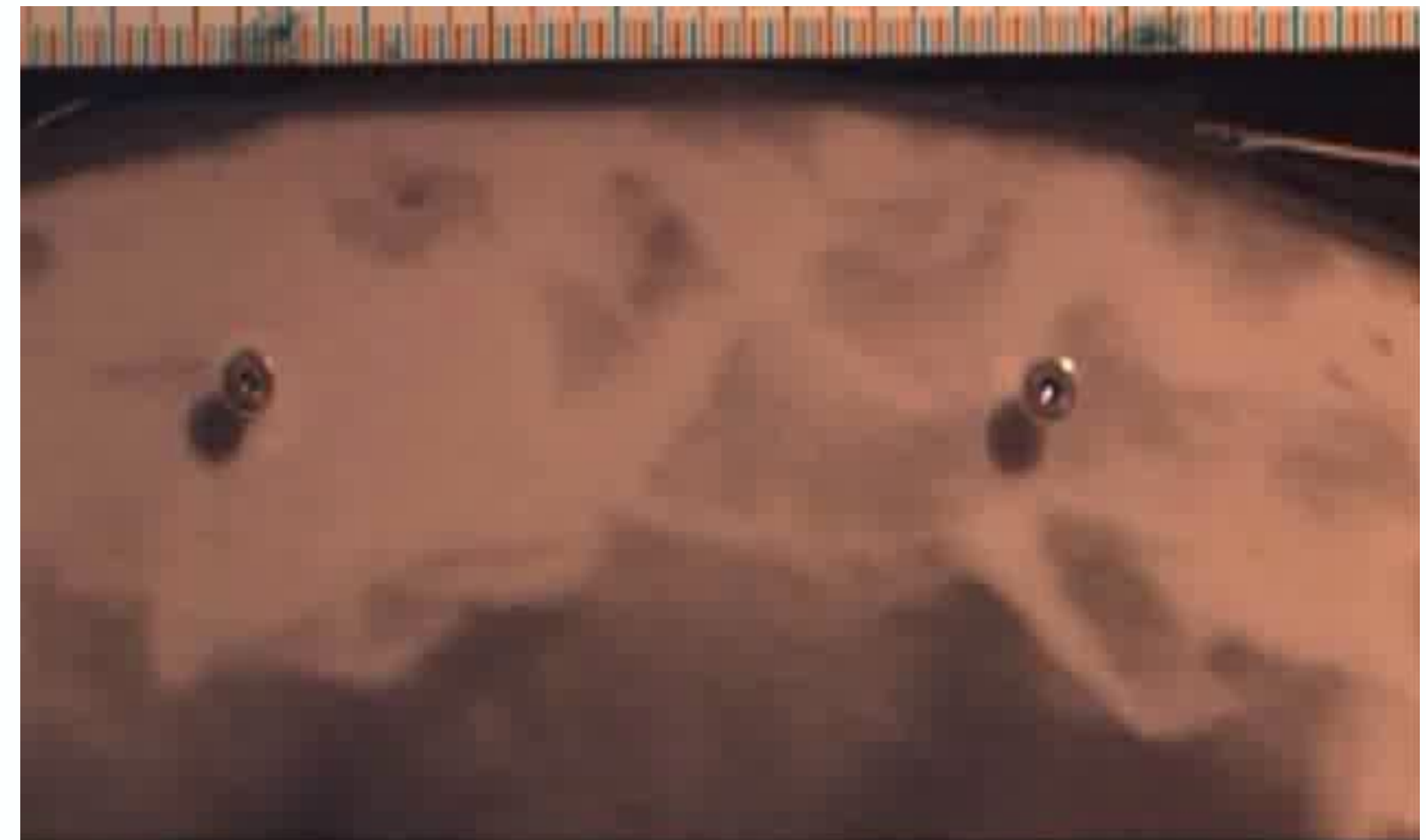

12:00:12 AM 0001 -0000.2[ms] S01 [5000 Hz] SpeedCam MiniVis

1.6 mm particles (10000 FPS)

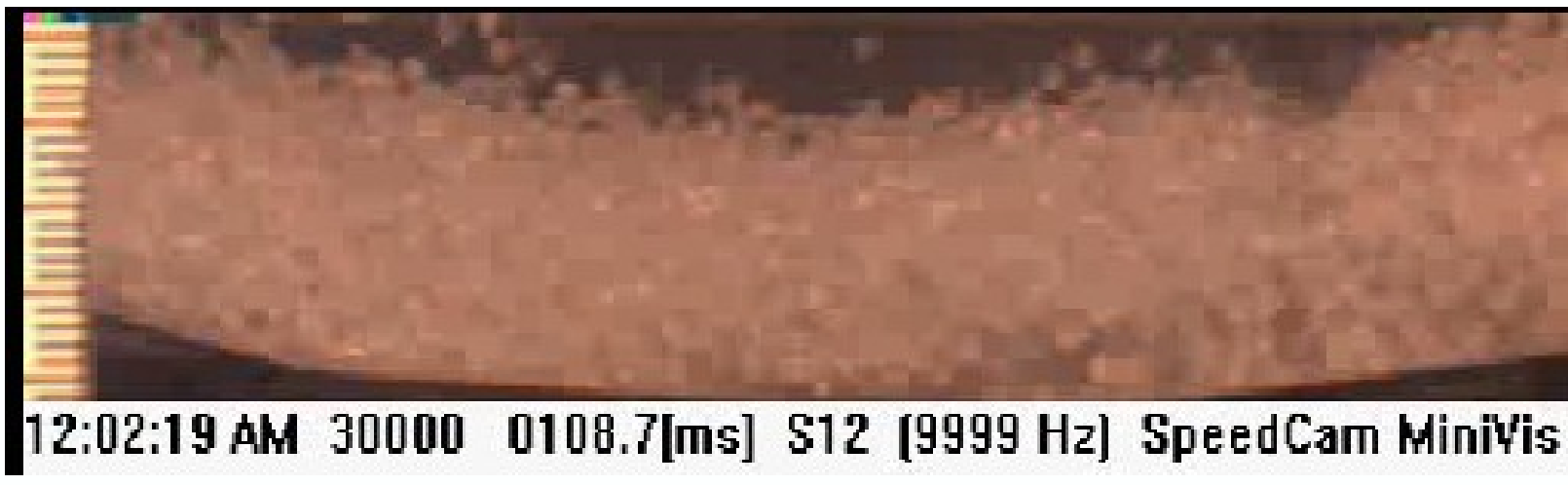




\section{Non-reacting Flow Modeling}




\section{Computational Fluid Dynamics}

- Computational fluid dynamics $\rightarrow$ Fluent 13.0

- Eulerian/Eulerian two-fluid model, granular solid phase

- Gidaspow drag model ${ }^{1}$

$$
\begin{aligned}
& \left\{\begin{array}{l}
\beta=150 \frac{\alpha_{\mathrm{p}}\left(1-\alpha_{\mathrm{g}}\right) \mu_{\mathrm{g}}}{\alpha_{\mathrm{g}} d_{\mathrm{p}}^{2}}+1.75 \frac{\rho_{\mathrm{g}} \alpha_{\mathrm{p}}\left|\vec{u}_{\mathrm{p}}-\vec{u}_{\mathrm{g}}\right|}{d_{\mathrm{p}}} \text { for } \alpha_{\mathrm{g}} \leq 0.8 \\
\beta=\frac{3}{4} C_{\mathrm{D}} \frac{\alpha_{\mathrm{p}} \alpha_{\mathrm{g}} \rho_{\mathrm{g}}\left|\vec{u}_{\mathrm{p}}-\vec{u}_{\mathrm{g}}\right|}{d_{\mathrm{p}}} \alpha_{\mathrm{g}}^{-2.65} \text { for } \alpha_{\mathrm{g}}>0.8
\end{array}\right. \\
& C_{\mathrm{D}}= \begin{cases}\frac{24}{\operatorname{Re}_{\mathrm{p}}}\left(1+0.15 \operatorname{Re}_{\mathrm{p}}^{0.687}\right) & \left(\operatorname{Re}_{\mathrm{p}} \leq 1000\right) \\
0.44 & \left(\operatorname{Re}_{\mathrm{p}}>1000\right)\end{cases}
\end{aligned}
$$

Model geometries tested:

(a)

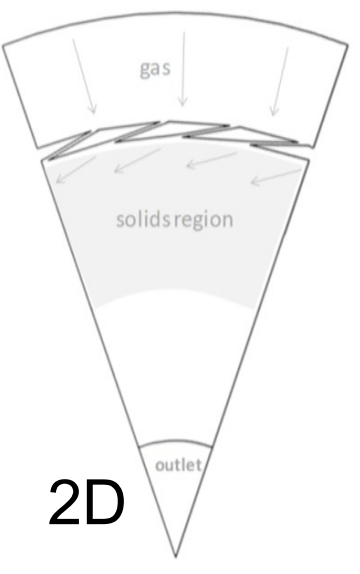

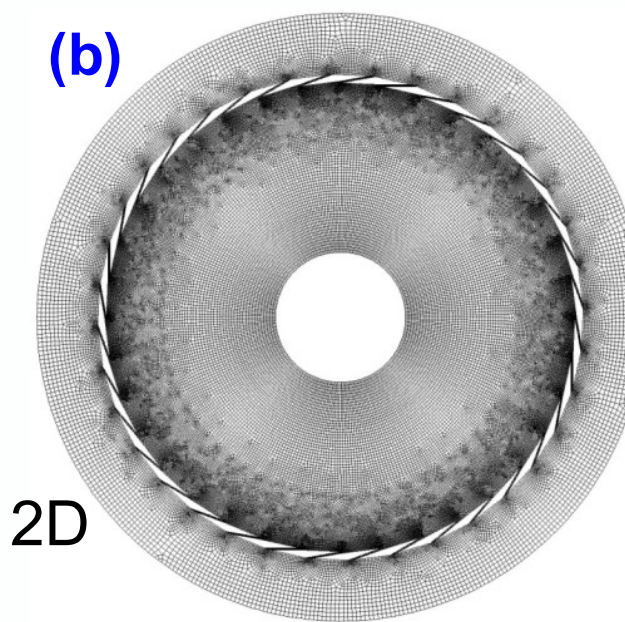

(c)

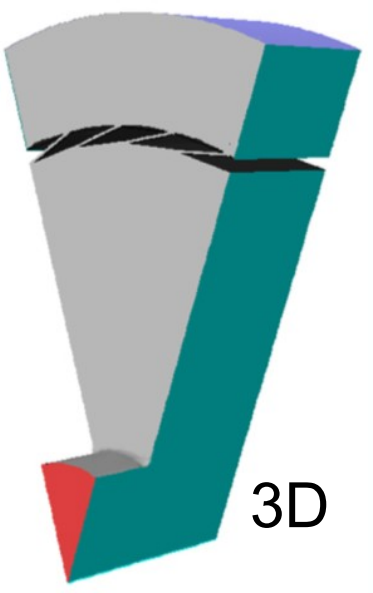

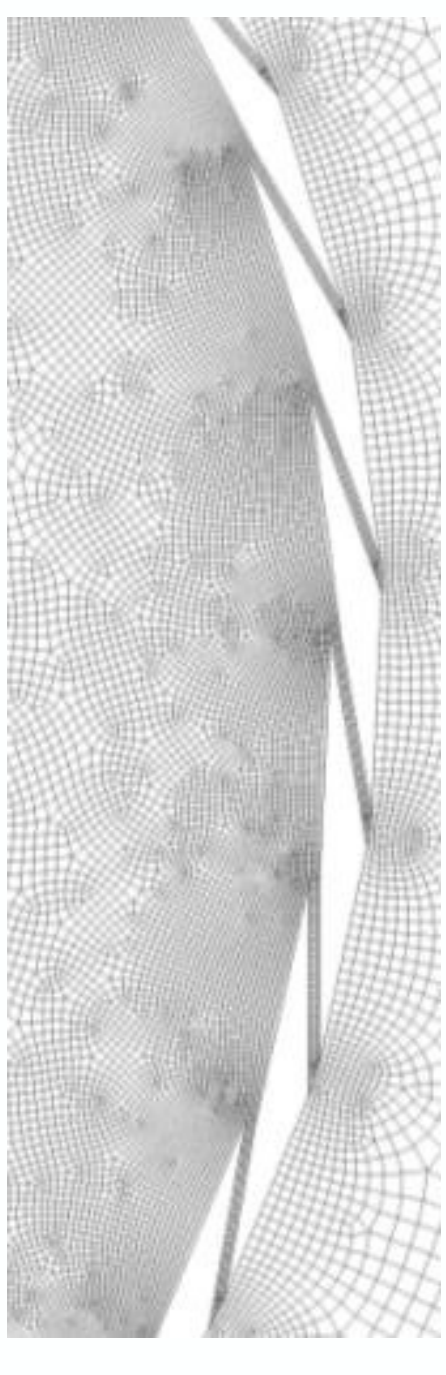




\section{General Non-reacting Flow Results}

Bed Mass: $2.1 \mathrm{~kg}-4.4 \mathrm{~kg}$

Air Flow Rate: $0.5 \mathrm{~kg} / \mathrm{s}-1.0 \mathrm{~kg} / \mathrm{s}$

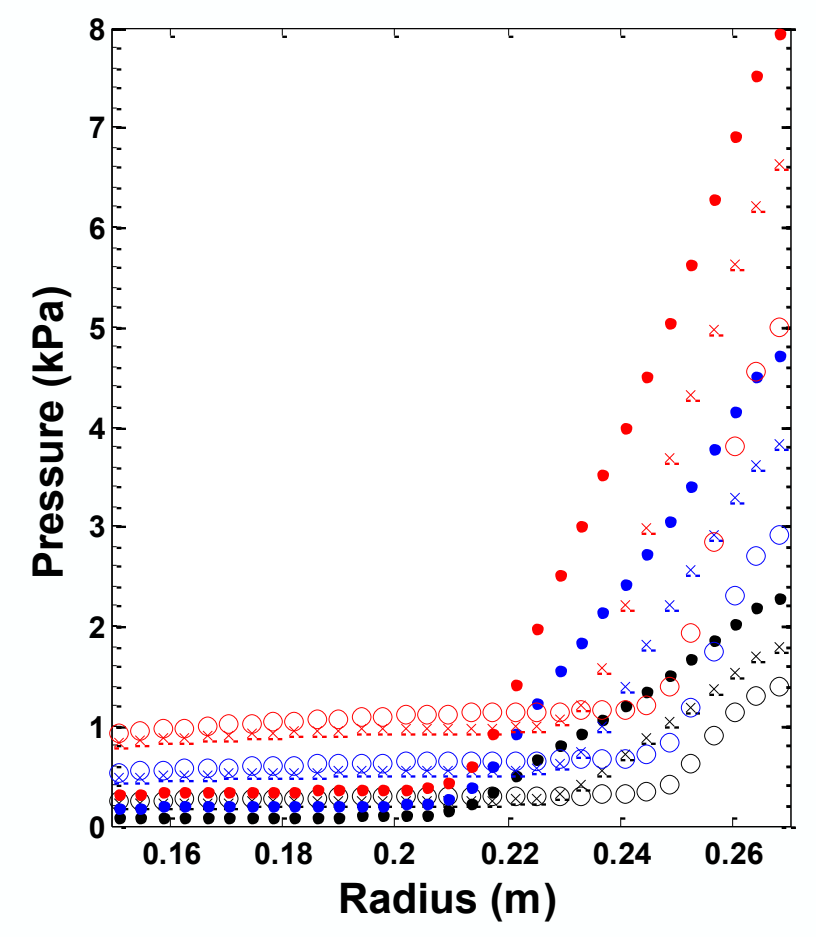

Bed $\Delta P: 2 \mathrm{kPa}-8 \mathrm{kPa}$

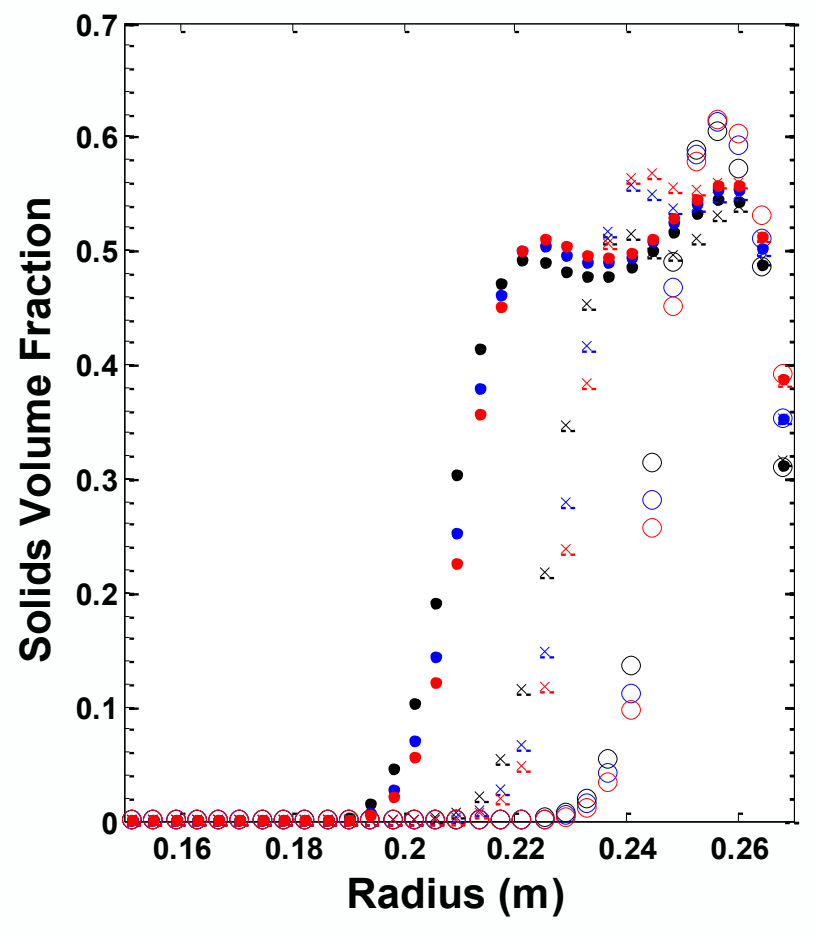

Solids VF: $0.4-0.6$

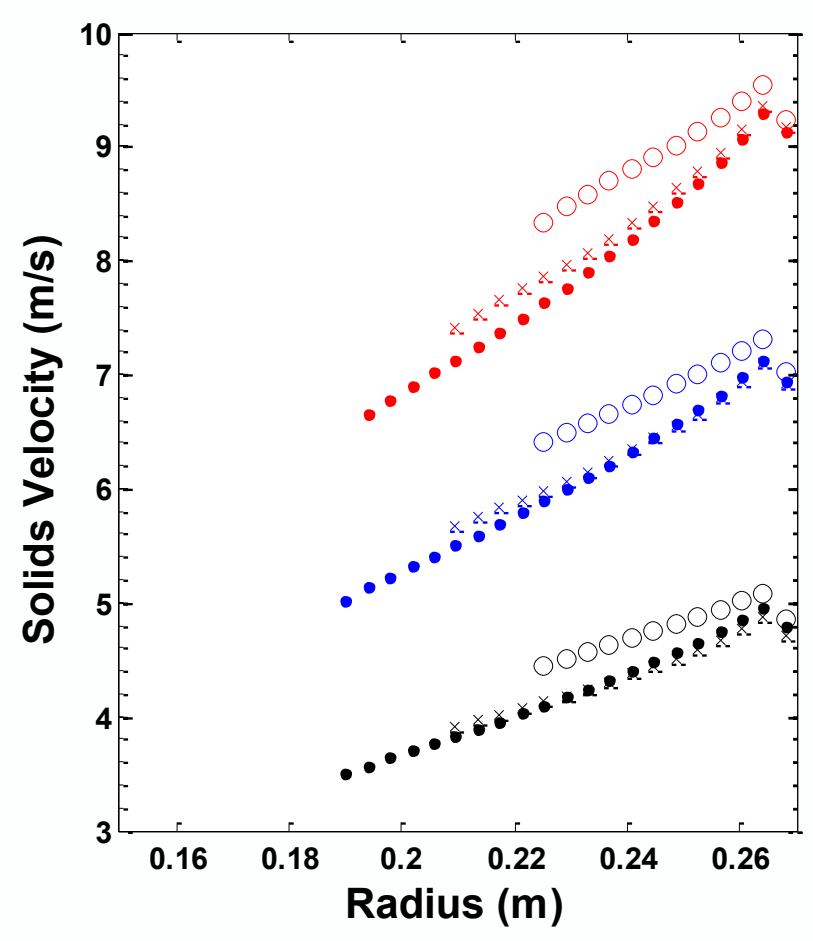

Solids Velocity: $4-9 \mathrm{~m} / \mathrm{s}$ 


\section{CFD Example Movies}

2D (with gravity), $0.74 \mathrm{~kg} / \mathrm{s}$ air, $3250 \mathrm{~g}$ bed

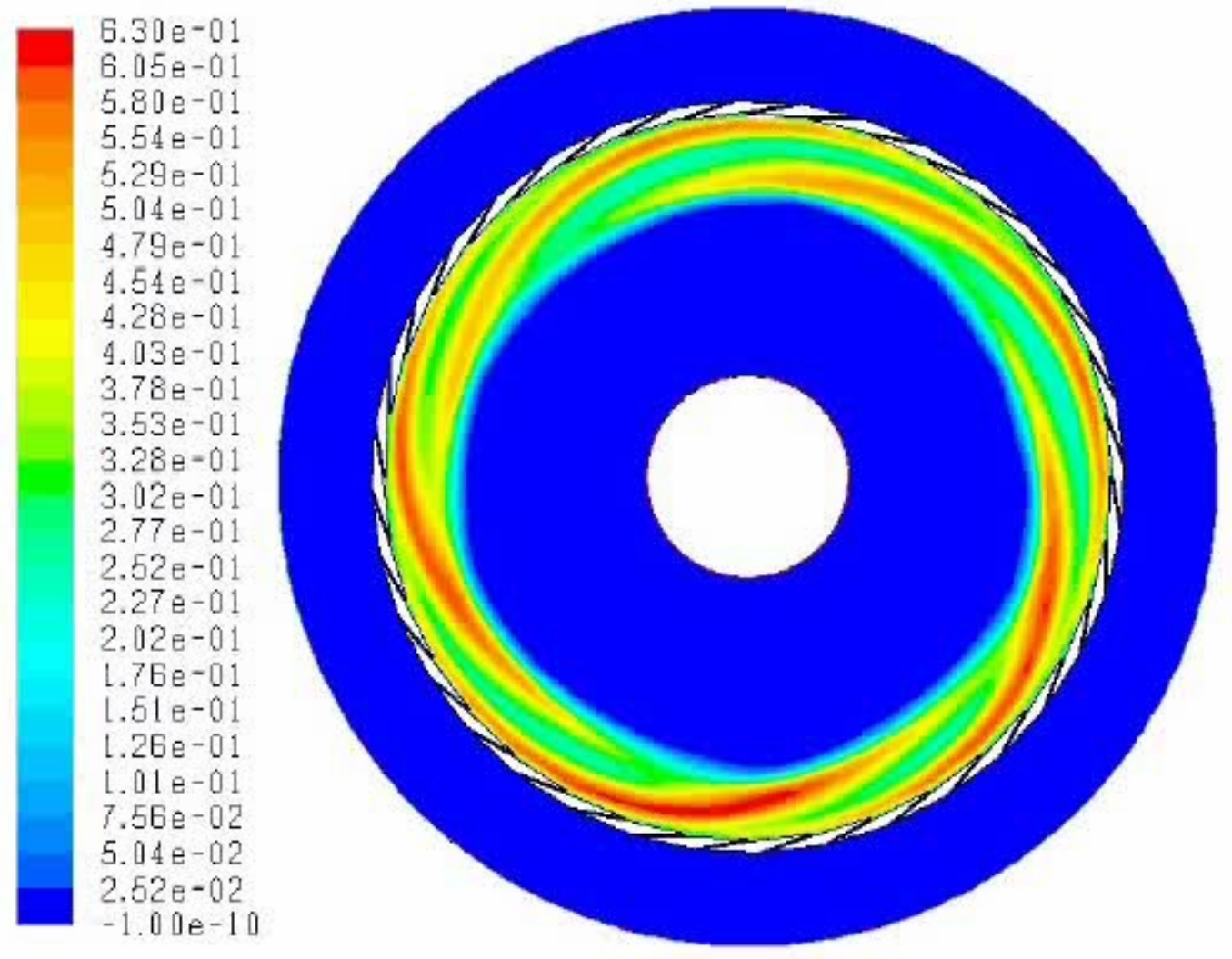

Contours of Volume fraction (polymer) (Time=2.0608e+01) ANSYS FLUENT 13.0 (2d, pbns, eulerian, rke, transient) 


\section{CFD Example Movies}

3D, $0.74 \mathrm{~kg} / \mathrm{s}$ air, $3250 \mathrm{~g}$ bed (iso-surfaces $=0.40$ and 0.01 solids volume fraction)
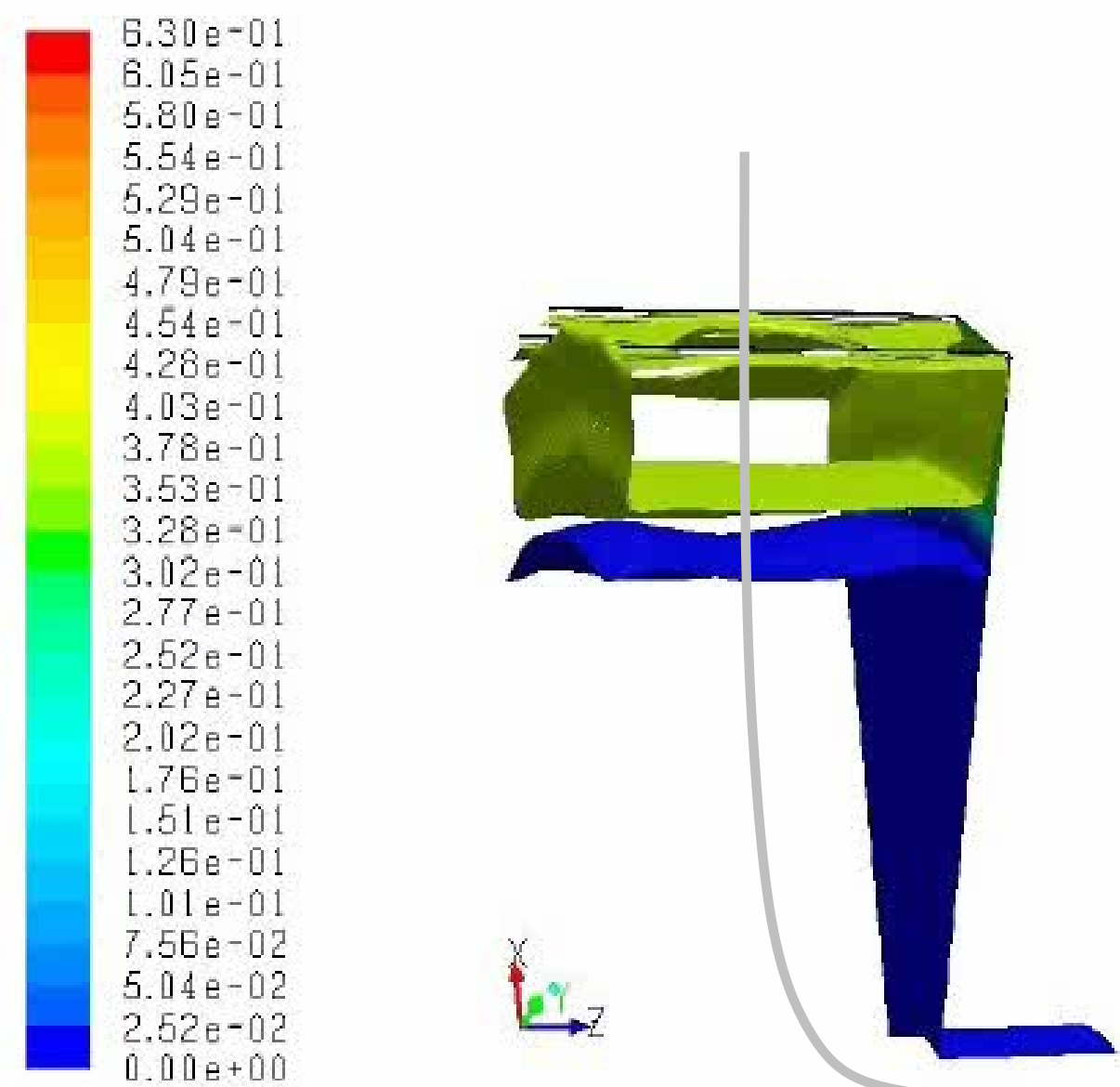

Contours of Volume fraction (polymer) (Time=1.3002e+01) ANSYS FLUENT 13.0 (3d, pbns, eulerian, rke, transient) 


\section{Model Validation}




\section{Bed Pressure Drop and Bed Thickness}

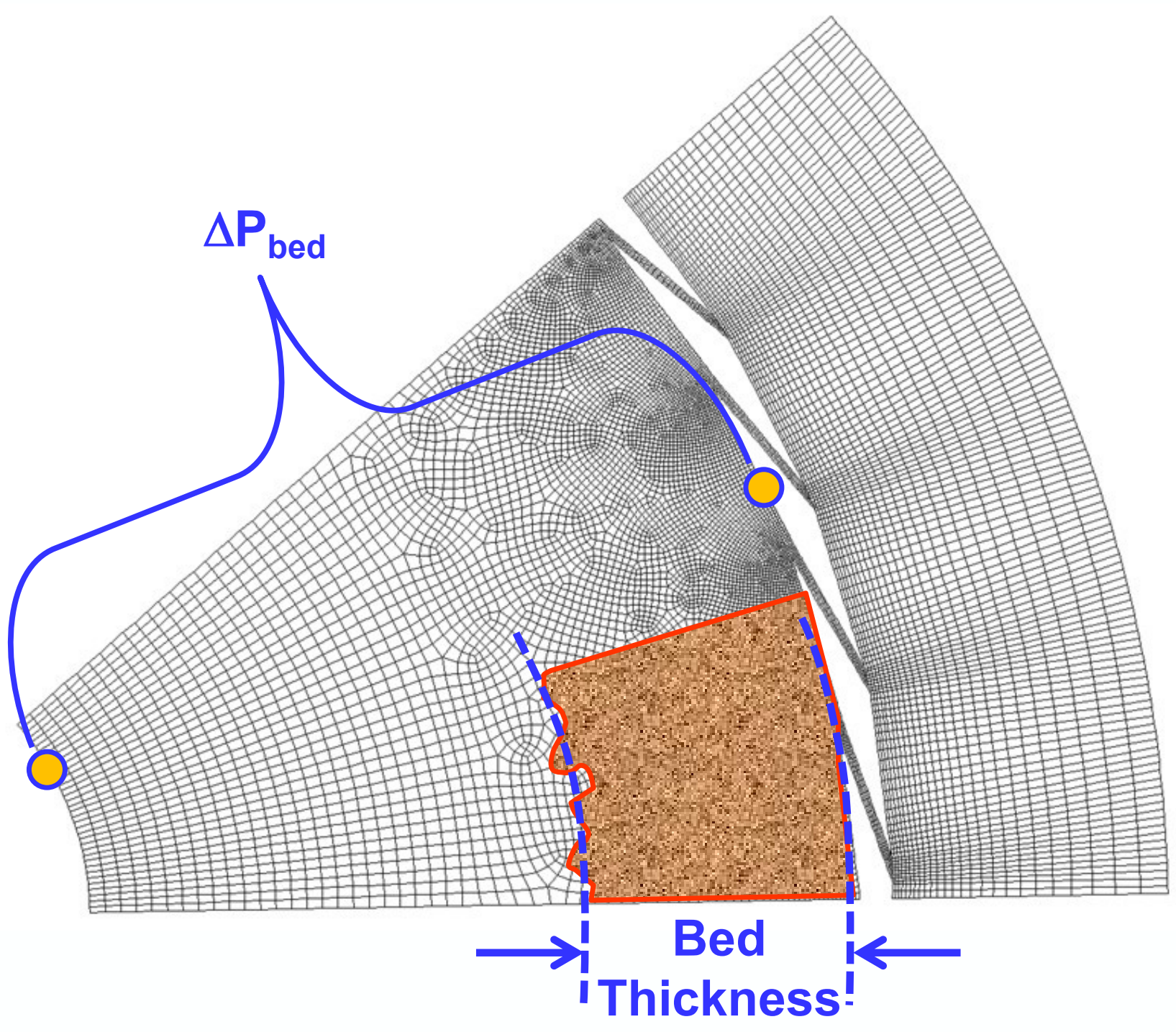

Pressure Field (Pa)

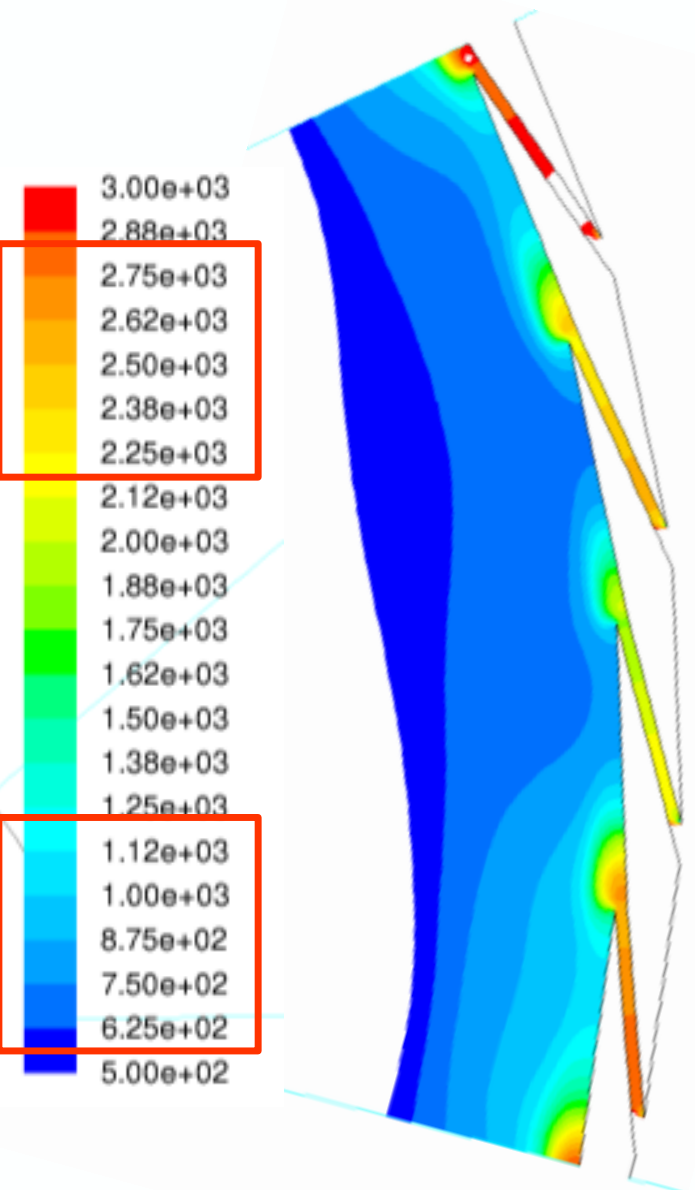




\section{Bed and Bed Thickness (2D)}

Air flow: $\quad 0.5 \mathrm{~kg} / \mathrm{s} \quad 0.75 \mathrm{~kg} / \mathrm{s} \quad 1.0 \mathrm{~kg} / \mathrm{s}$

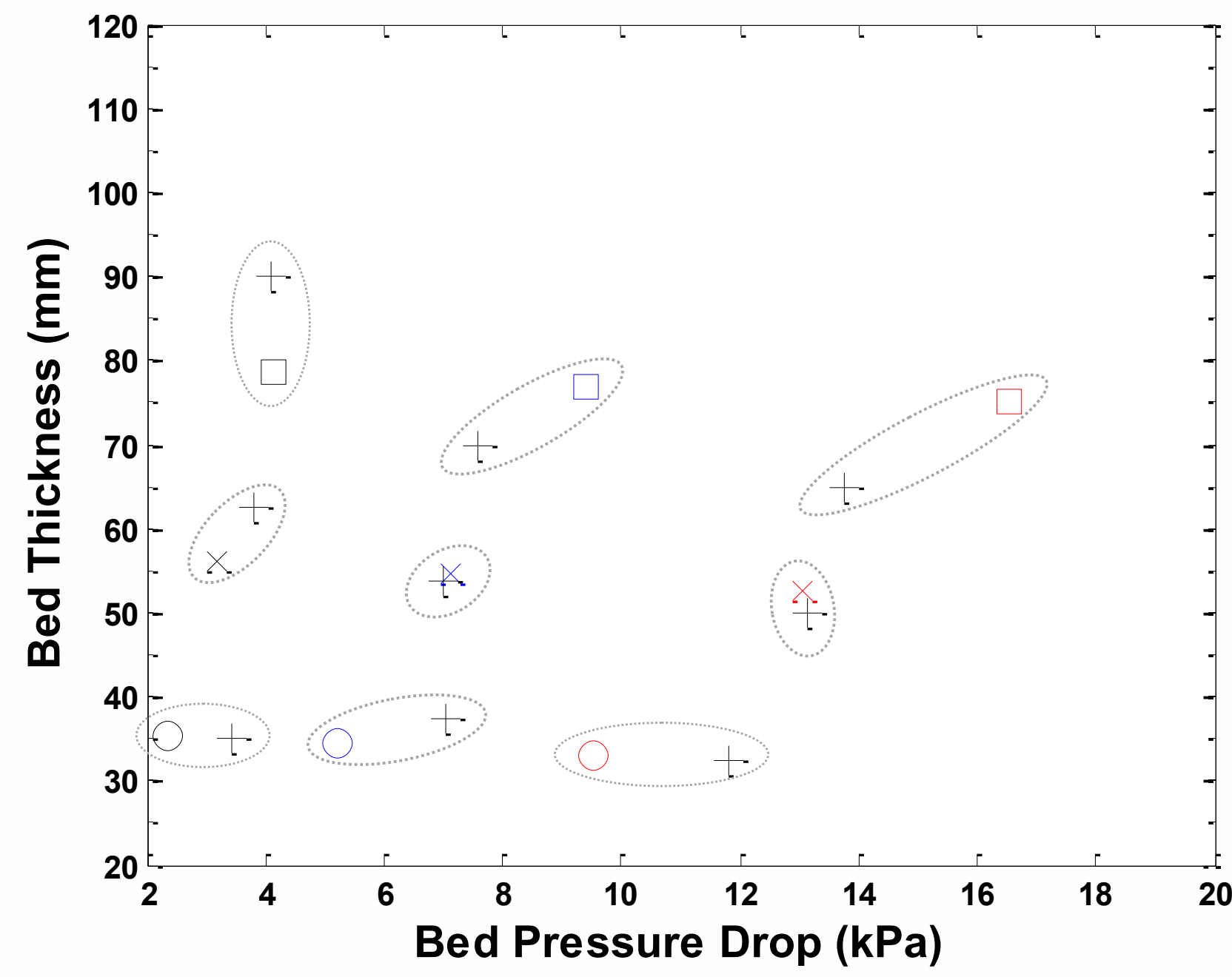

$4.38 \mathrm{~kg}$ bed mass

$3.25 \mathrm{~kg}$ bed mass

$2.12 \mathrm{~kg}$ bed mass

Experimental data: $(+)$ signs 


\section{Model Deficiencies \& Refinement}

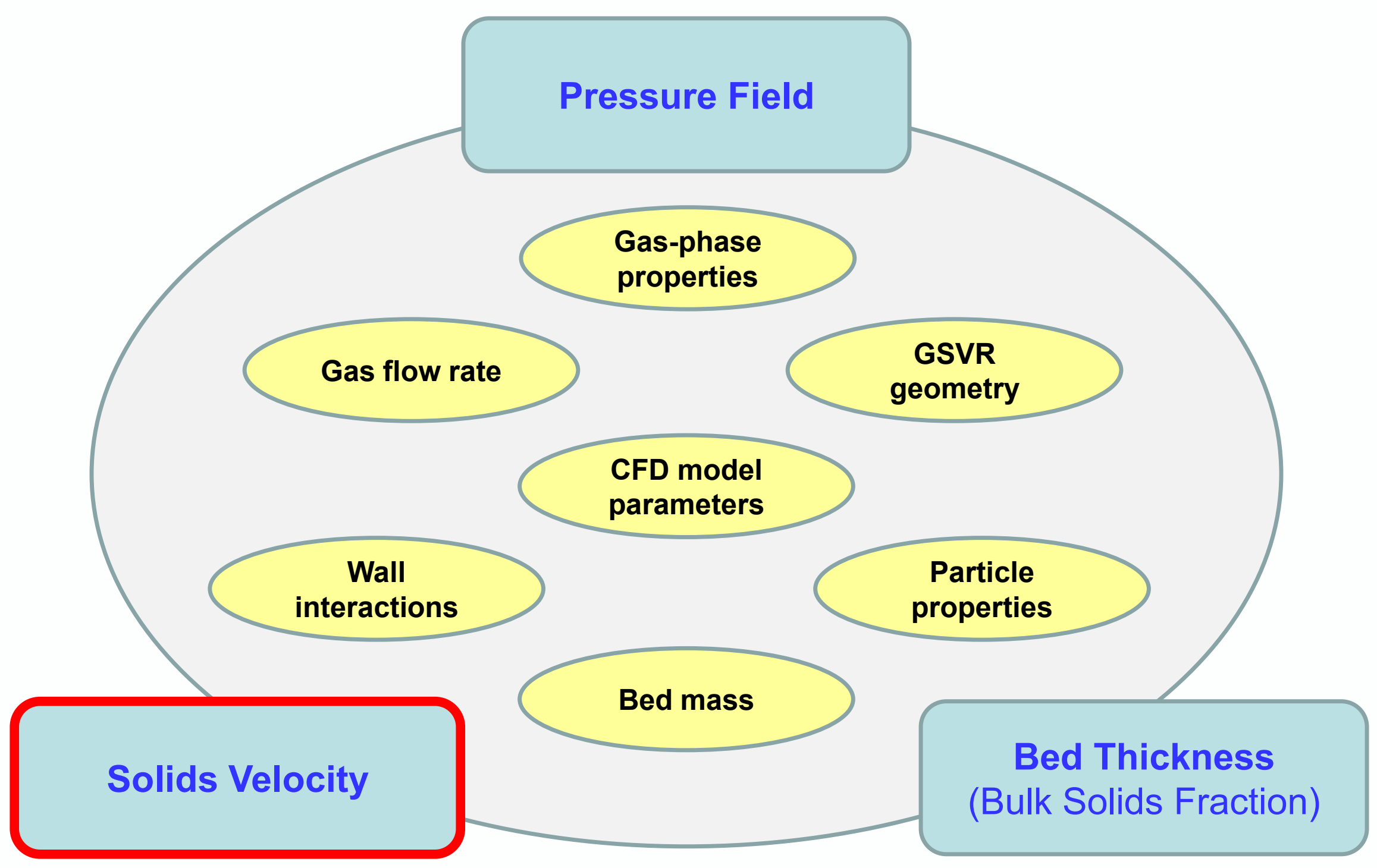




\title{
Particle Image Velocimetry (PIV)
}

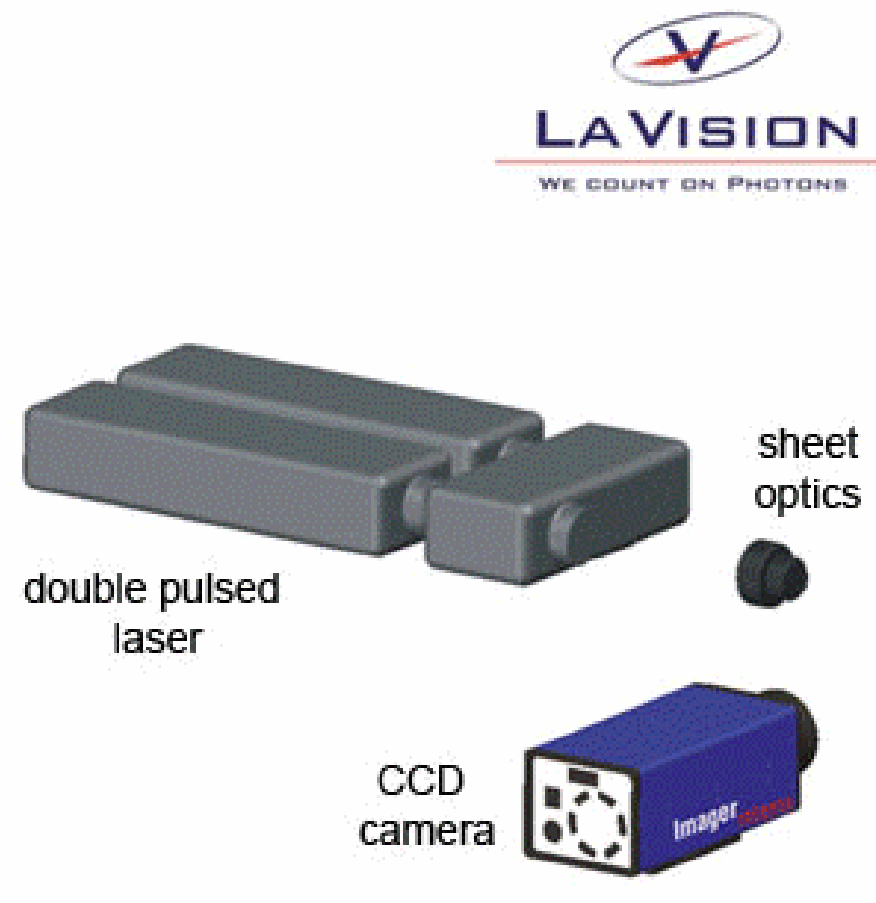

\author{
flow \\ direction
}
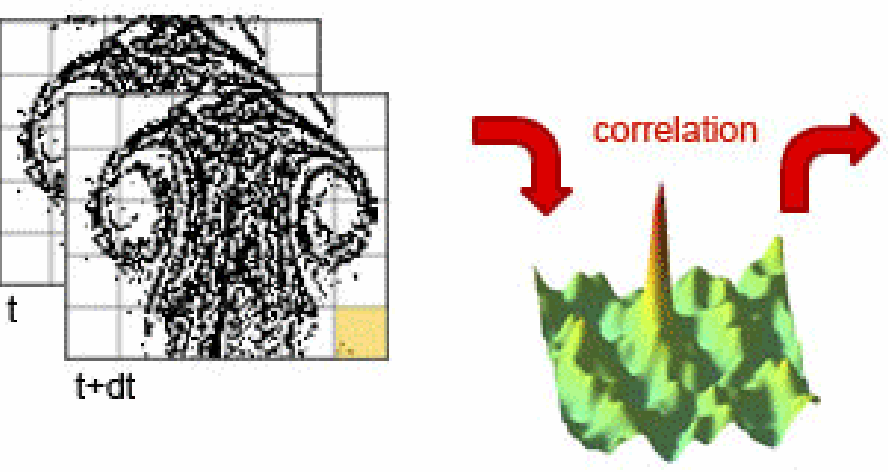

\begin{tabular}{|l|l|l|l|l|l|}
\hline. & 1 & 1 & 1 &, &. \\
\hline- & 1 & $\uparrow$ & $\uparrow$ & 1 &. \\
\hline & 1 & $\uparrow$ & $\uparrow$ & 1 &, \\
\hline. &. & 1 & 1 &, & - \\
\hline. &. & 1 & 1 &. &. \\
\hline
\end{tabular}

La Vision --- http://www.piv.de/piv/index.php 


\section{Modeling Biomass Pyrolysis}




\section{Biomass Pyrolysis in a GSVR}

Traditional Static Fluidzed Bed ${ }^{1}$

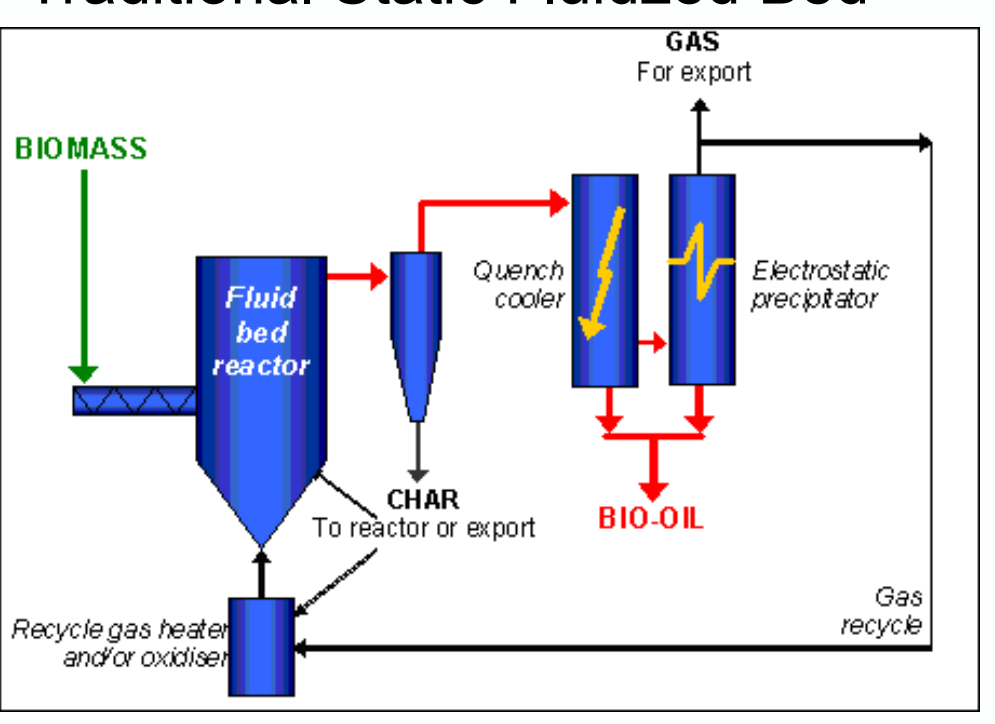

\section{GSVR}

$\mathrm{D}=54 \mathrm{~cm}$ (solids region)

$\mathrm{L}=10 \mathrm{~cm}$

\section{Biomass} feed zone $(300 \mathrm{~K})$

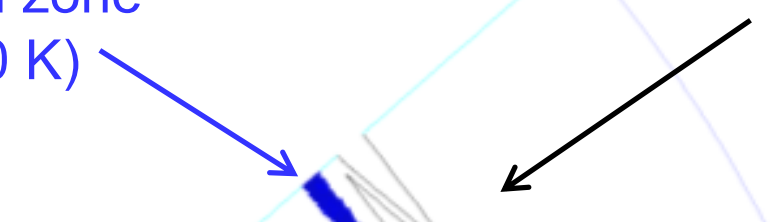

Products
$\mathrm{N}_{2}(923 \mathrm{~K})$

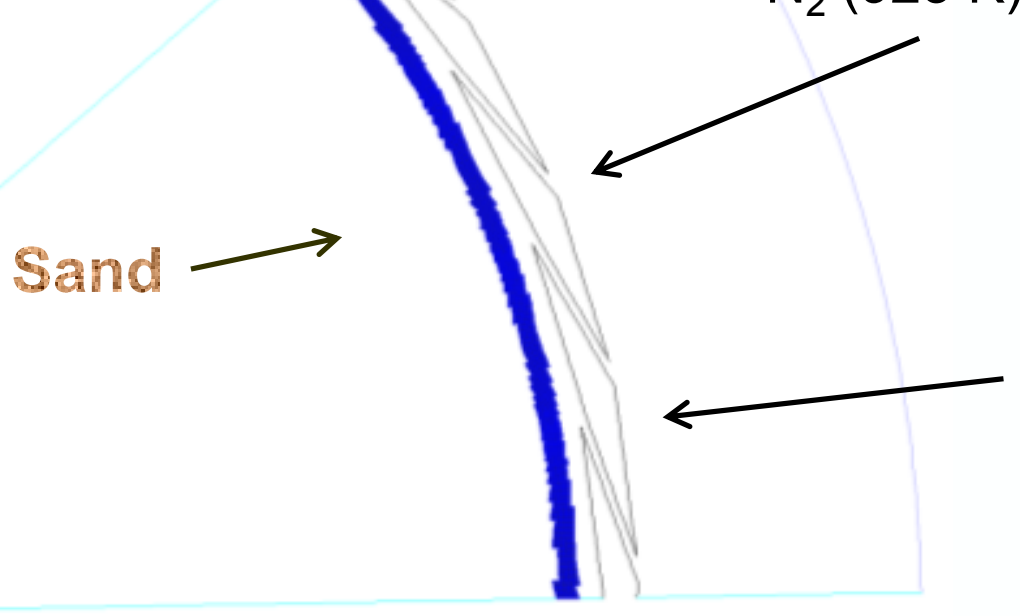




\section{Pyrolysis Modeling in a GSVR}

Volume fraction

- 2D periodic GSVR simulations

- Heterogeneous reactions (solid $\rightarrow$ gas + char):

- 10-reaction network with psuedo-components 1

- Continuous feeding of biomass

- Cellulose, hemicellulose, and lignin

- Different rates for each biomass component

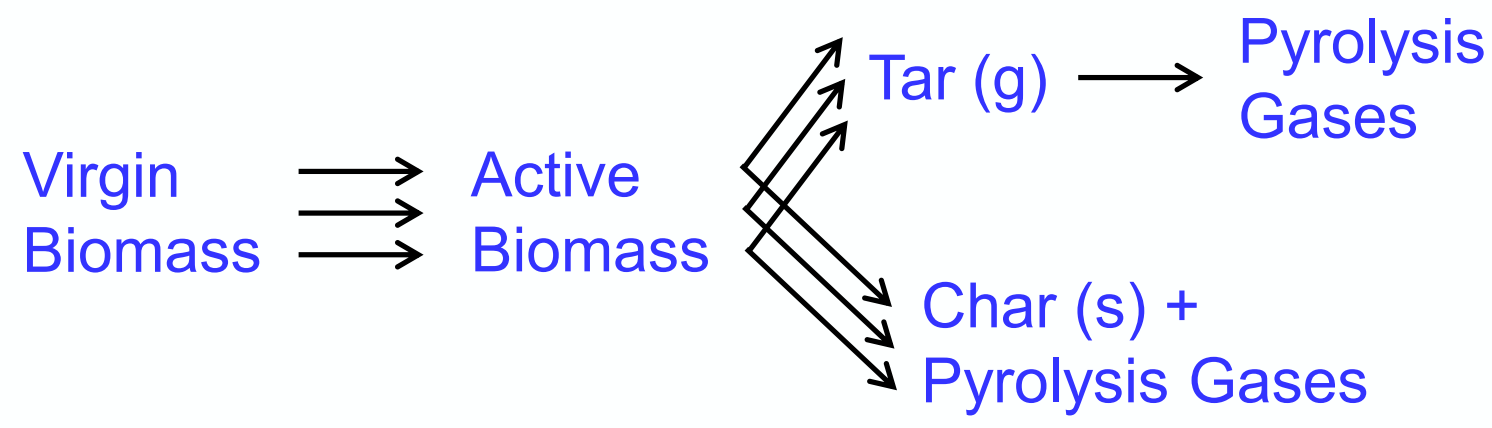

- 4-phase Eulerian multiphase simulation (3 granular)

- Gas, biomass, char, and sand

- Sand and biomass retained in reactor

- Char leaves with gas flow due to lower density
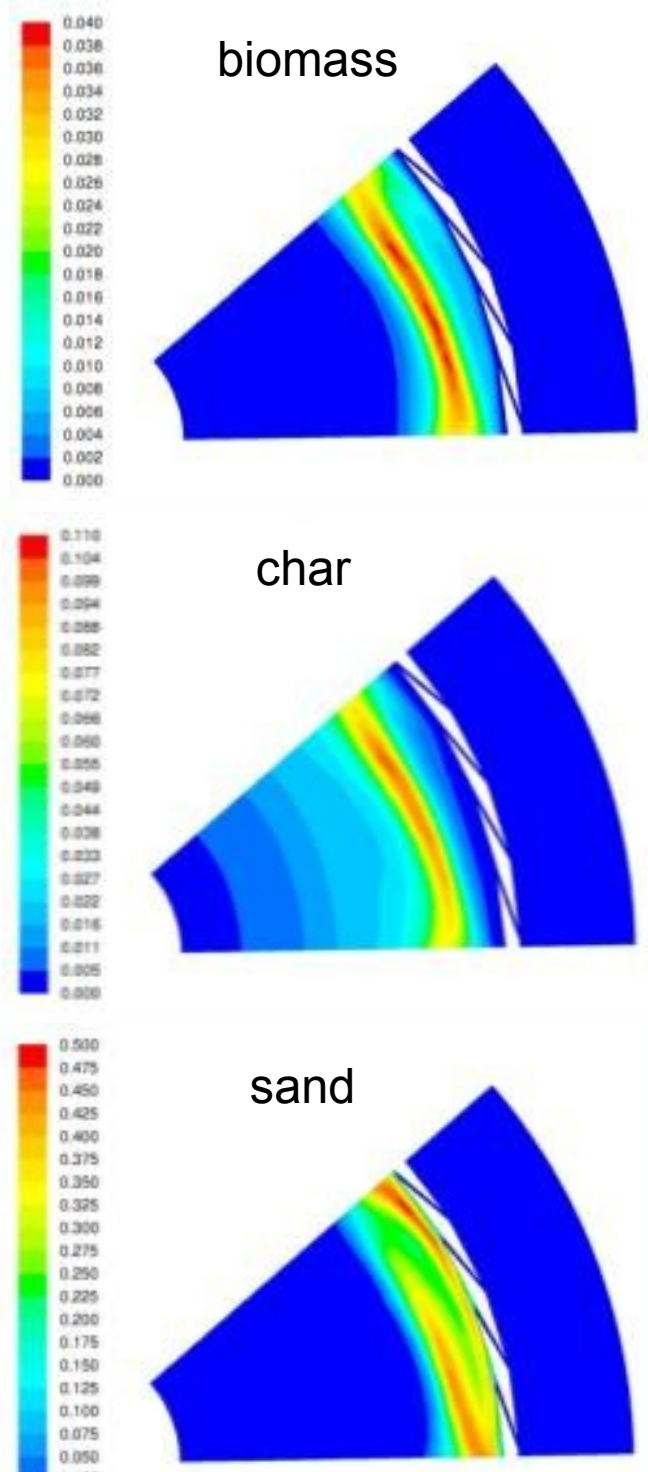


\section{Base GSVR Operating Variables}

Volume $\left(\mathrm{m}^{3}\right)$

0.023

Gas flow rate $(\mathrm{kg} / \mathrm{s})$

0.22

Biomass feed rate $(\mathrm{kg} / \mathrm{s})$

0.035

Biomass moisture content ( $w t \%$, dry basis)

10

Sand mass in reactor $(\mathrm{kg})$

5

Gas-to-biomass ratio $\left(\mathrm{kg}_{\text {gas }} / \mathrm{kg}_{\text {biomass }}\right)$

6.4

Gas feed temperature (K)

923

Biomass feed temperature $(K)$

300

Biomass feed rate/volume $\left(\mathrm{kg} / \mathrm{m}^{3} \cdot \mathrm{s}\right)$

1.5

$\rightarrow$ Biomass composition (wt $\%$ dry):

- $36 \%$ cellulose

- $47 \%$ hemicellulose

- $17 \%$ lignin 


\section{Volume Fraction and Temperature}

(a)

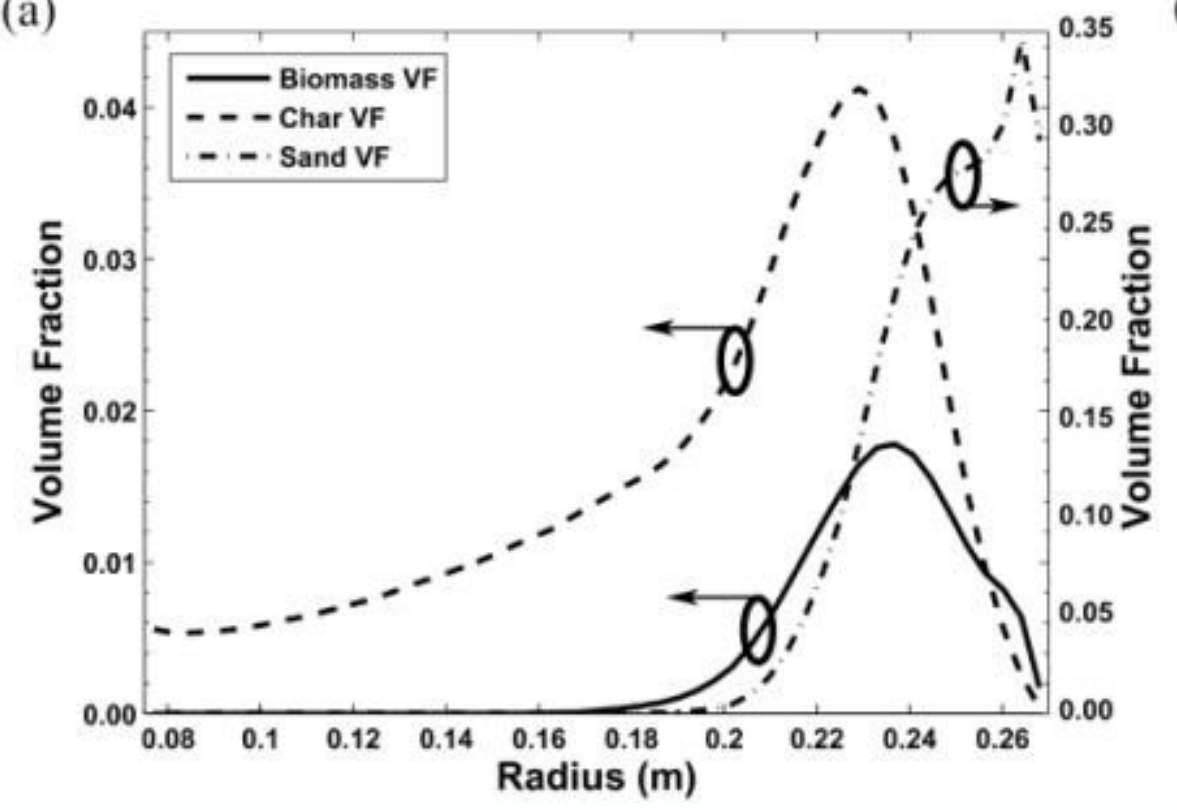

(b)

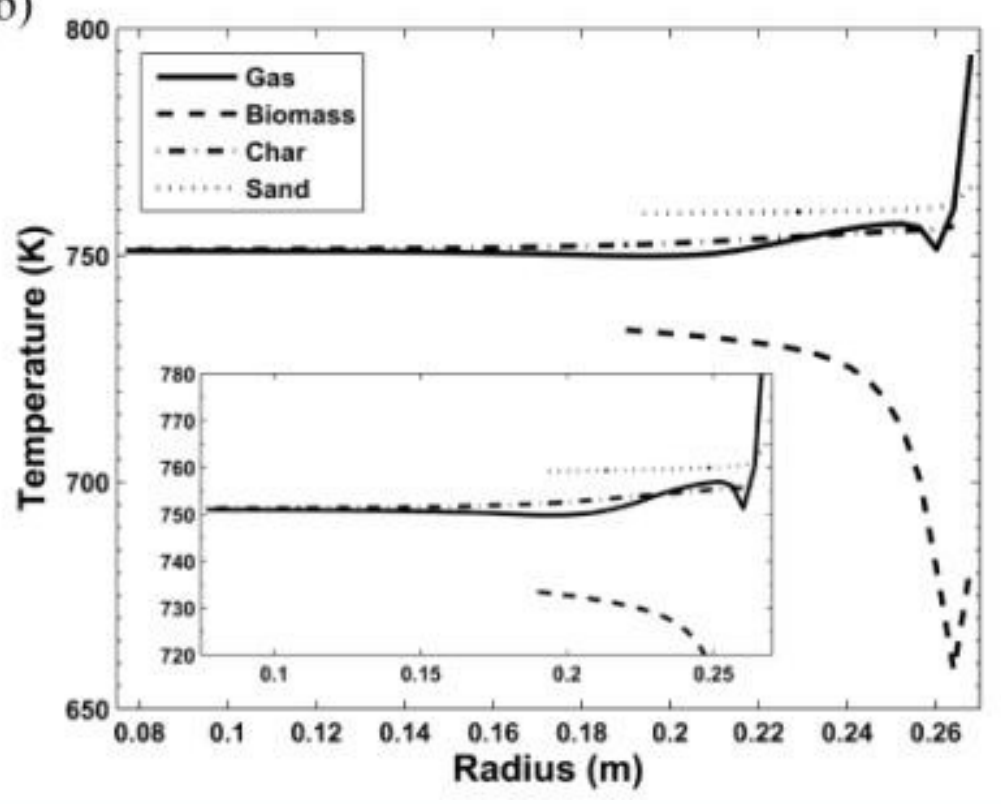

\subsection{0}

0.030
0.029
0.027
0.025
0.024
0.022
0.021
0.020
0.018
0.016
0.015
0.013
0.012
0.010
0.009
0.007
0.006
0.004
0.003
0.002
0.000

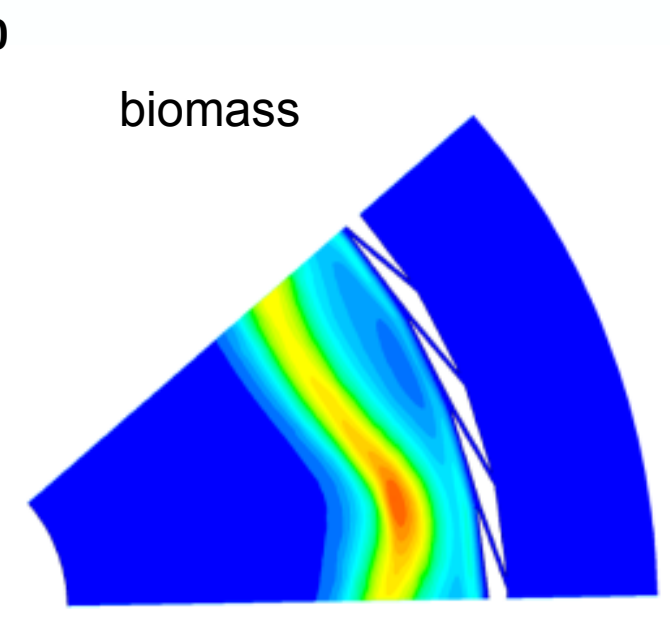

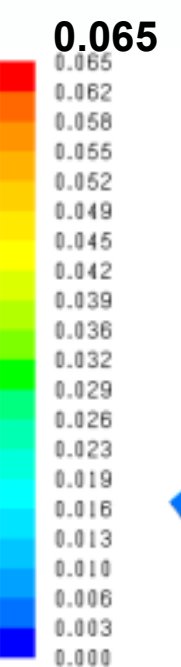

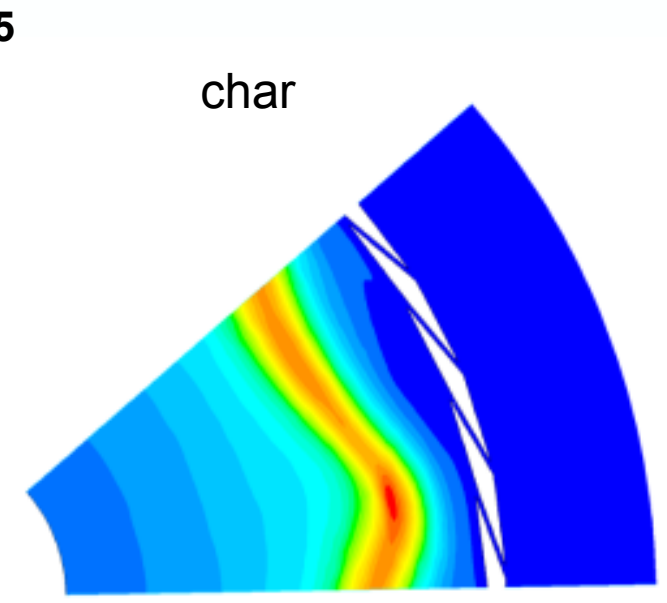

0.55

0.550
0.523
0.495
0.468
0.440
0.413
0.385
0.358
0.330
0.303
0.275
0.248
0.220
0.193
0.165
0.138
0.110
0.083
0.055
0.027
0.000

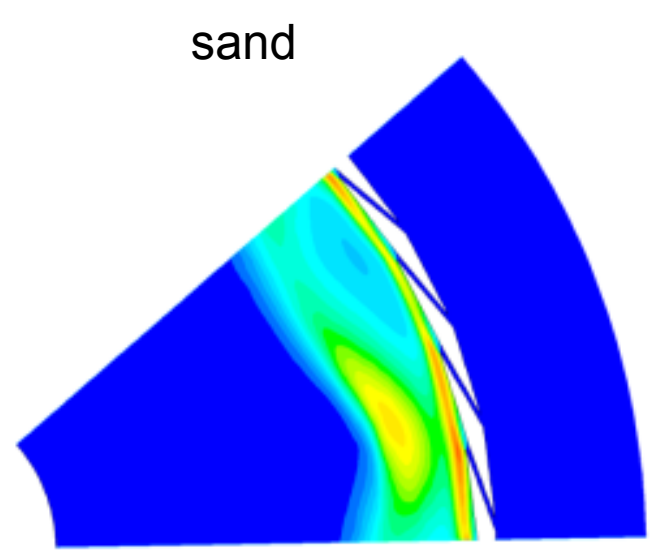

27 


\section{Volume Fraction Animation}

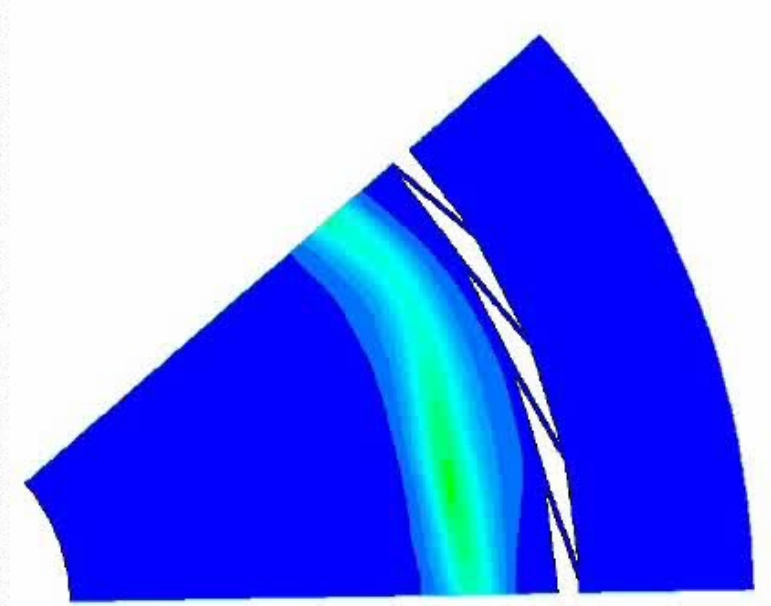

\subsection{5}

$1.50 e-01$
$1.43 e-01$

1. $35 e-01$

$1.27 e-01$

$1.13 e-01$

1.05e-01

$9.75 e-0 z$

$9.00 e-02$

$8.25 \mathrm{e}-02$

$7.50 \mathrm{e}-02$

$6.75 e-02$

$6.00 e-02$

$5.25 e-02$

$4.50 \mathrm{e}-02$

$3.75 e-02$

$3.00 e-02$

$2.25 e-02$

$1.50 e-02$

$7.50 e-03$

$4.00 e-03$

$0.00 e+00$

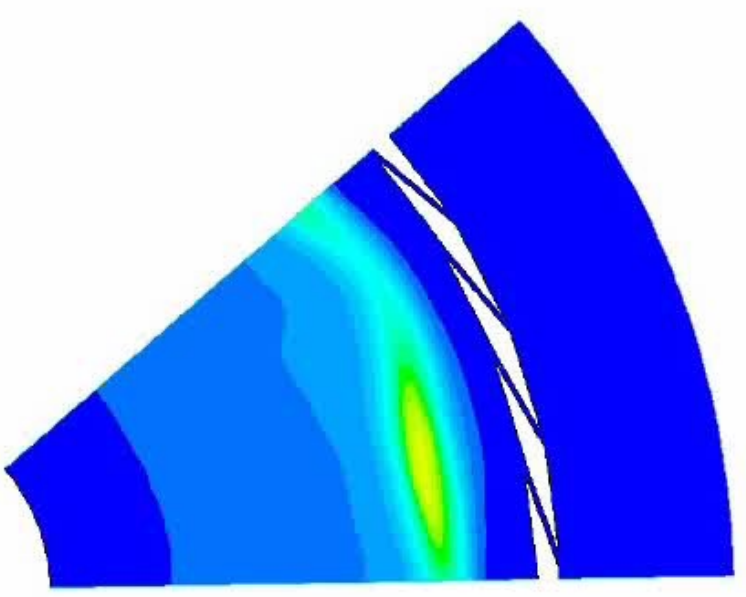

$\nearrow$

\subsection{3}

Biomass

\section{6. $30 \mathrm{e}-01$}

$5.99 e-0$

$5.67 e-01$
$5.35 e-01$

$5.35 e-0$.
$5.04 e-0$.

$4.72 \mathrm{e}-01$

$4.41 e-01$

$4.10 \mathrm{e}-0$

$3.78 \mathrm{e}-01$

$3.47 e-01$

$3.15 e-01$

$2.83 e-01$

$2.52 e-01$

$2.20 e-01$

$1.89 e-01$

$1.57 e-01$

1.26e-01

9. $45 \mathrm{e}-02$

$6.30 e-02$

3. $15 e-02$

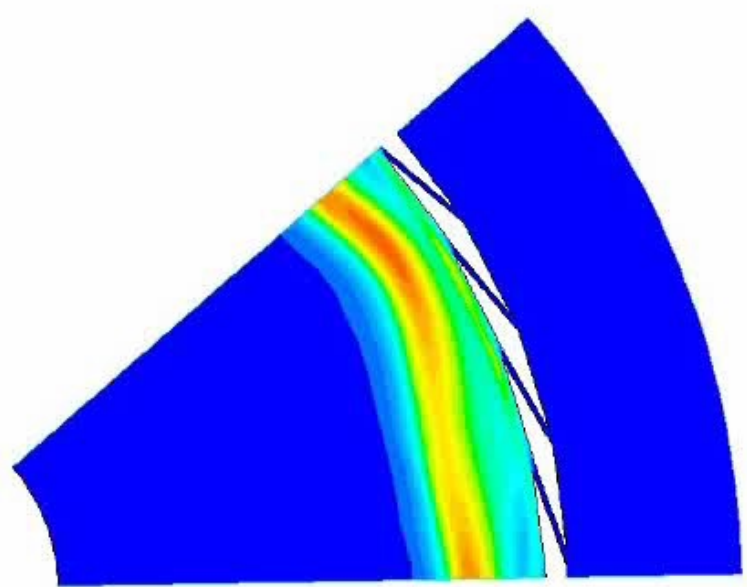

$\nwarrow$

Char

Total solids

(biomass, char, sand)

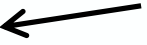




\section{Comparison - GSVR vs Fluidized Bed}

\section{Static Fluidized Bed ${ }^{1}$}

$$
\begin{aligned}
& D=3.81 \mathrm{~cm} \\
& \mathrm{H}=34.3 \mathrm{~cm}
\end{aligned}
$$

Products

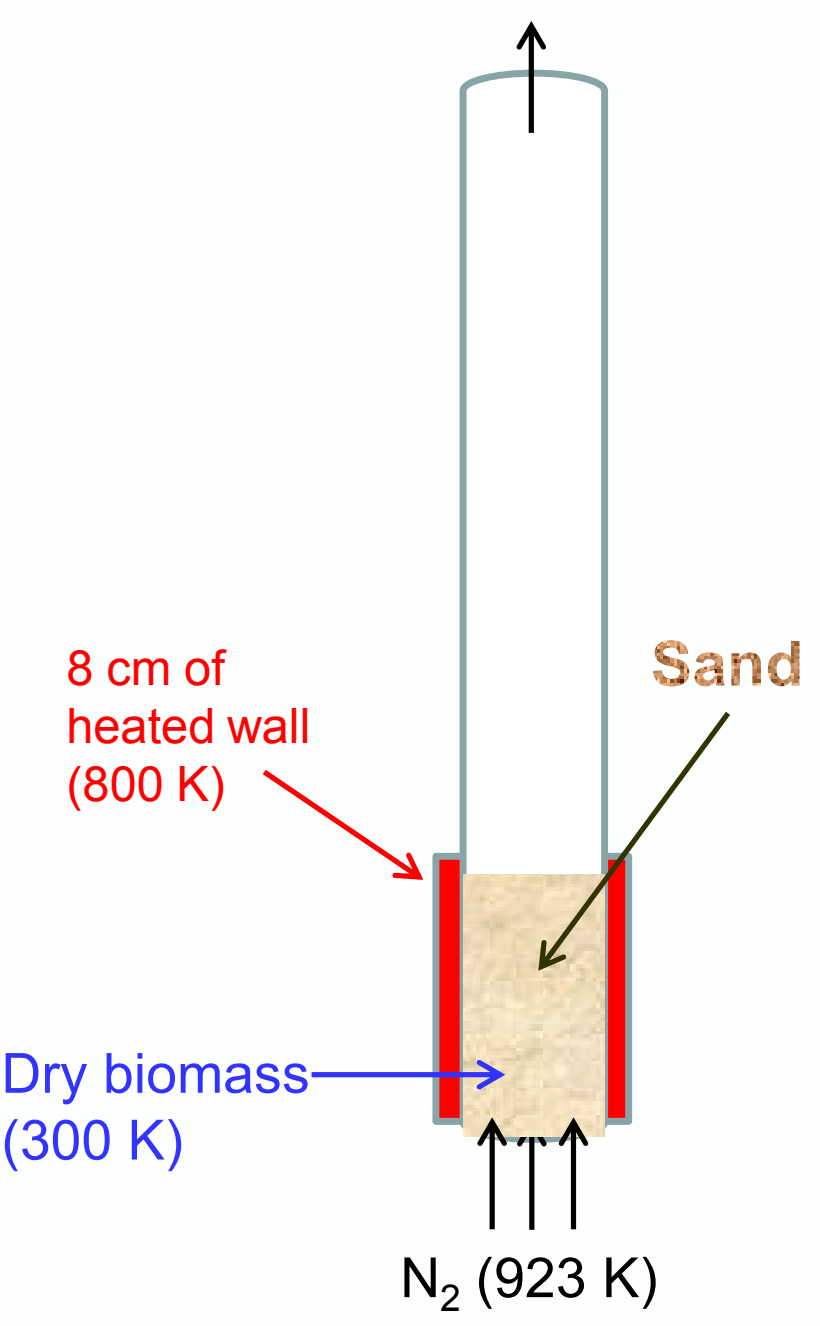

\section{GSVR}

$\mathrm{D}=54 \mathrm{~cm}$ (solids region)

$\mathrm{L}=10 \mathrm{~cm}$

Dry biomass

feed zone (300 K)

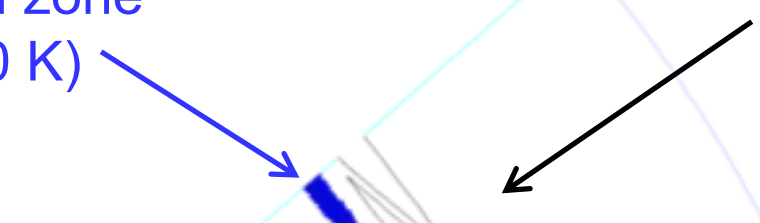

Products
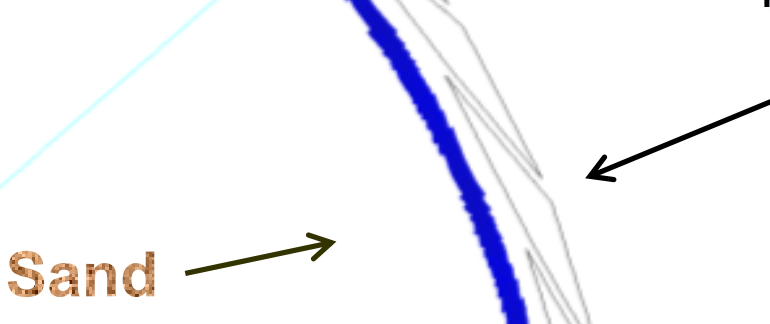

$\mathrm{N}_{2}(923 \mathrm{~K})$
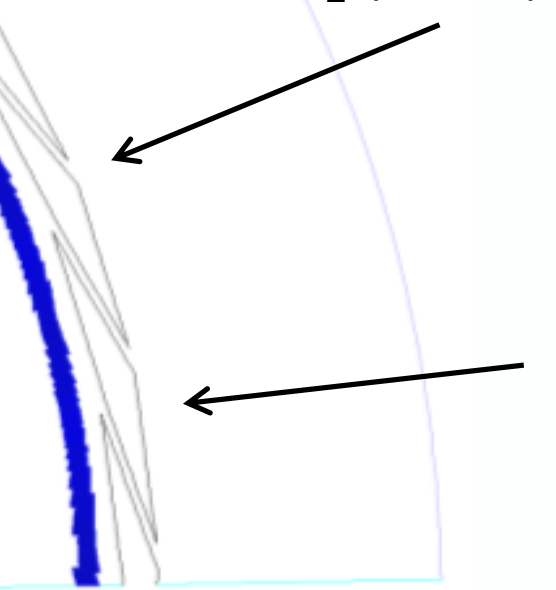


\section{Comparison - GSVR vs Fluidized Bed}

GSVR

Volume $\left(\mathrm{m}^{3}\right)$

Gas-to-biomass ratio $\left(\mathrm{kg}_{\text {gas }} / \mathrm{kg}_{\text {biomass }}\right)$

Sand mass in reactor/volume $\left(\mathrm{kg} / \mathrm{m}^{3}\right)$

Supplementary heating

Outlet Temperature (K)

Gas-phase residence time (s)

Product Yields (wt\% of fed biomass):

Tar

Pyrolysis gas

Char

Biomass (unconverted)
0.023

6.4

217

no

784

$\sim 0.05$

76.0

8.9

14.5

0.0

1.5

\section{Static FB ${ }^{1}$}

0.00039

6.4

322

yes

790

$\sim 0.75$

63.4

21.5

14.4

0.6

Biomass conversion rate / reactor volume $\left(\mathrm{kg} / \mathrm{m}^{3} \cdot \mathrm{s}\right)$ 
Process Intensification 


\section{GSVR Process Intensification}

Increase gas and biomass feed rates

\section{proportionately}

- Base feed rates:

$0.22 \mathrm{~kg} / \mathrm{s}$ gas

$0.035 \mathrm{~kg} / \mathrm{s}$ biomass

(a)

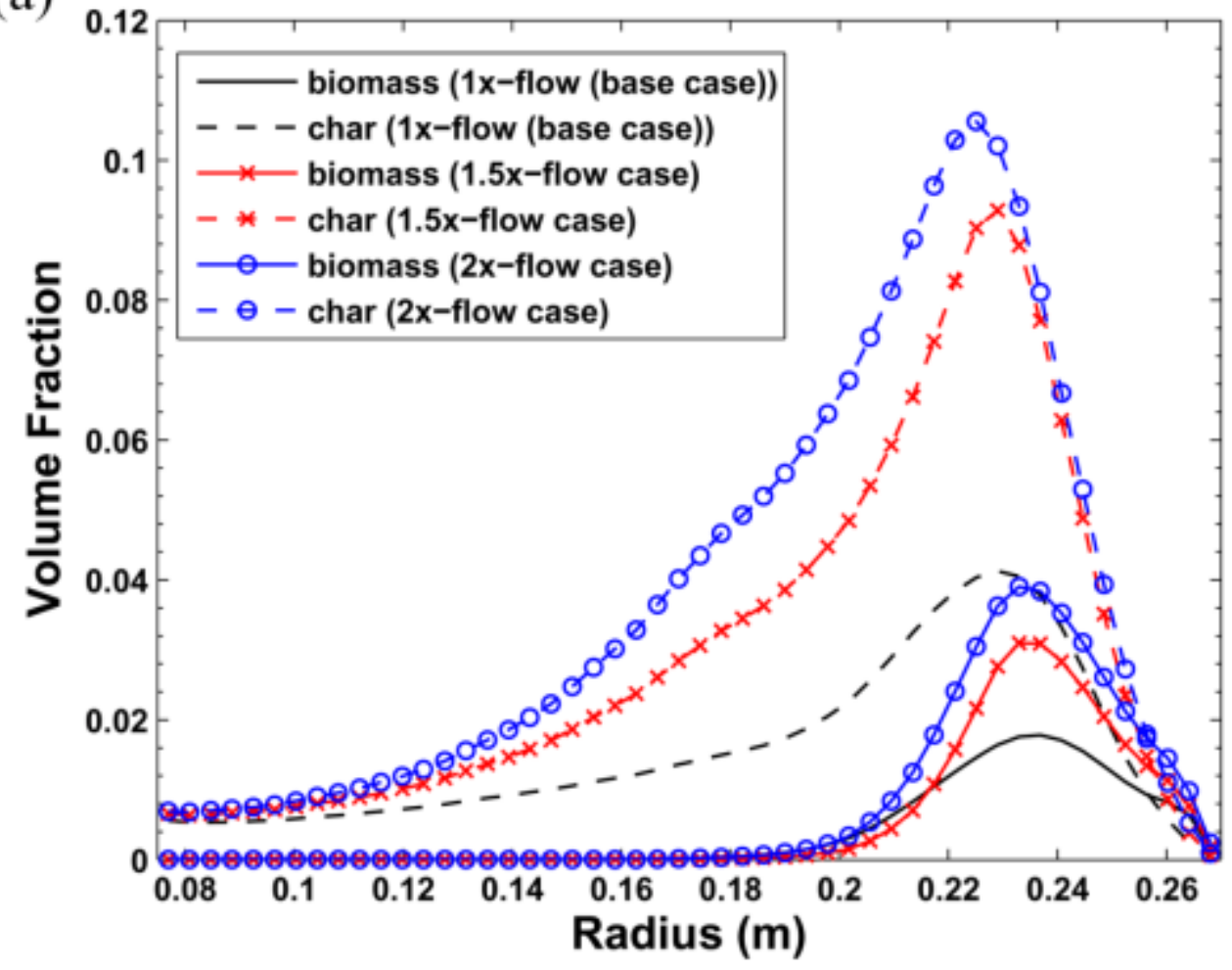

$$
\begin{aligned}
\mathrm{Nu}_{p}=\frac{h_{p} \cdot d_{p}}{\lambda_{g}}= & \left(7-10 \varepsilon_{g}+5 \varepsilon_{g}^{2}\right)\left(1+0.7 \cdot \operatorname{Re}_{p}^{0.2} \cdot \operatorname{Pr}^{\frac{1}{2}}\right) \\
& +\left(1.33-2.4 \varepsilon_{g}+1.2 \varepsilon_{g}^{2}\right) \cdot \operatorname{Re}_{p}^{0.7} \cdot \operatorname{Pr}^{\frac{1}{2}}
\end{aligned}
$$

(b)

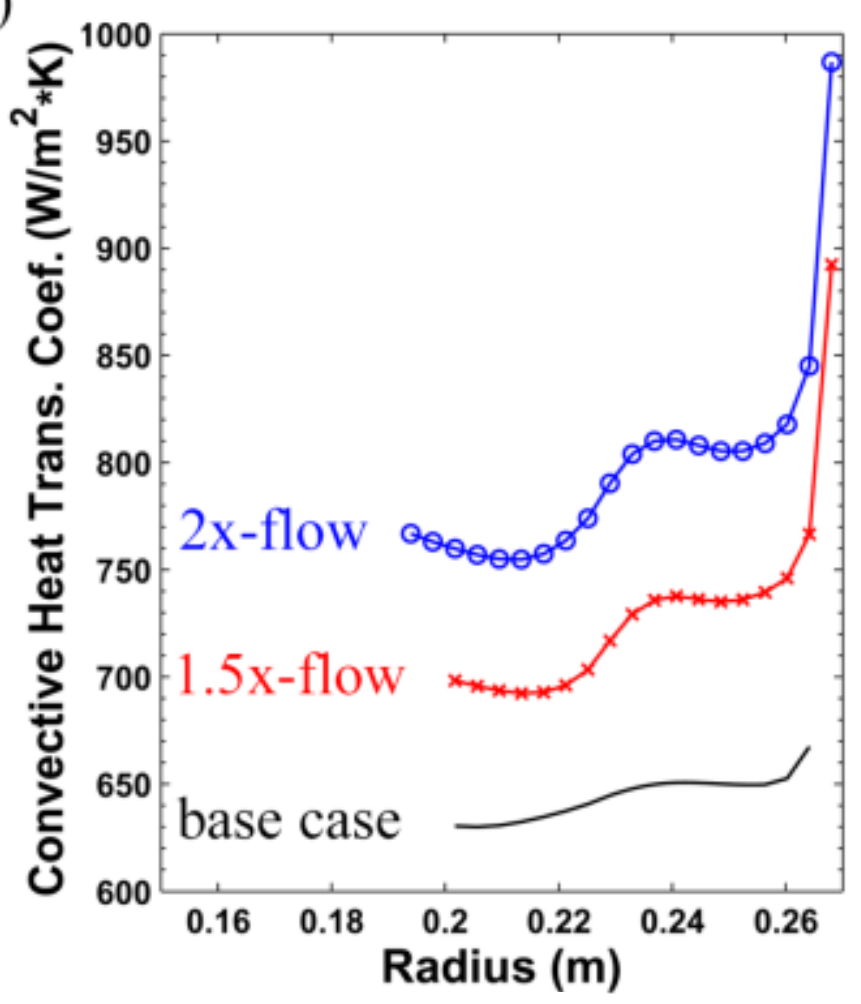

$\rightarrow$ Reactor performance and product yields $\sim$ the same (but increased $\Delta \mathrm{P}$ )

$\rightarrow$ Plus, shorter gas residence time and higher heat transfer coefficients 


\section{GSVR Process Intensification}

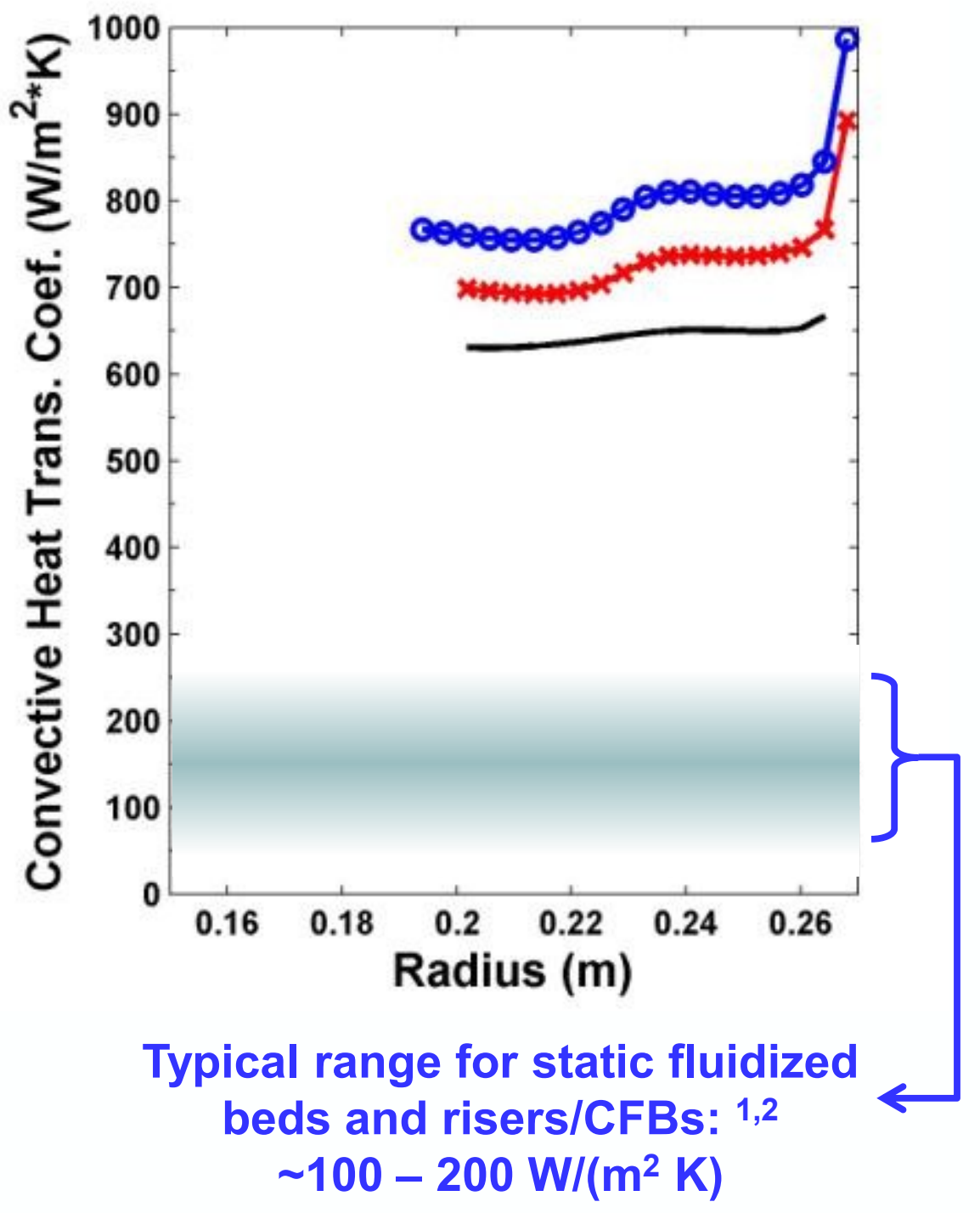

1. Z.Y. Zhou, A.B. Yu, P. Zulli, Particle scale study of heat transfer in ... fluidized beds, AIChE J. 55 (2009) 868-884

2. Y. Ma, J.X. Zhu, Experimental study of heat transfer in a co-current downflow fluidized bed, Chem. Eng. Sci. 54 (1999) 41-50 


\section{Pyrolysis Reactor without Sand}

Operating the reactor with biomass as the only solid

- Much larger char mass accumulates

- Product distribution the same as in cases with sand

- Char removal occurs in pulsing, oscillatory pattern

\section{Biomass}

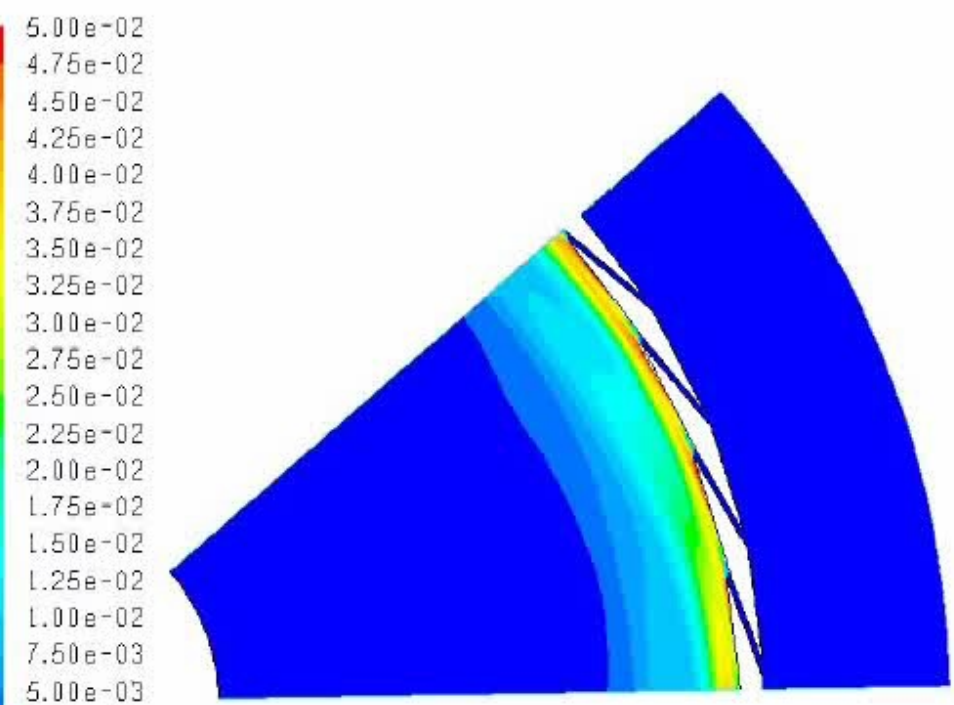

\section{Char}

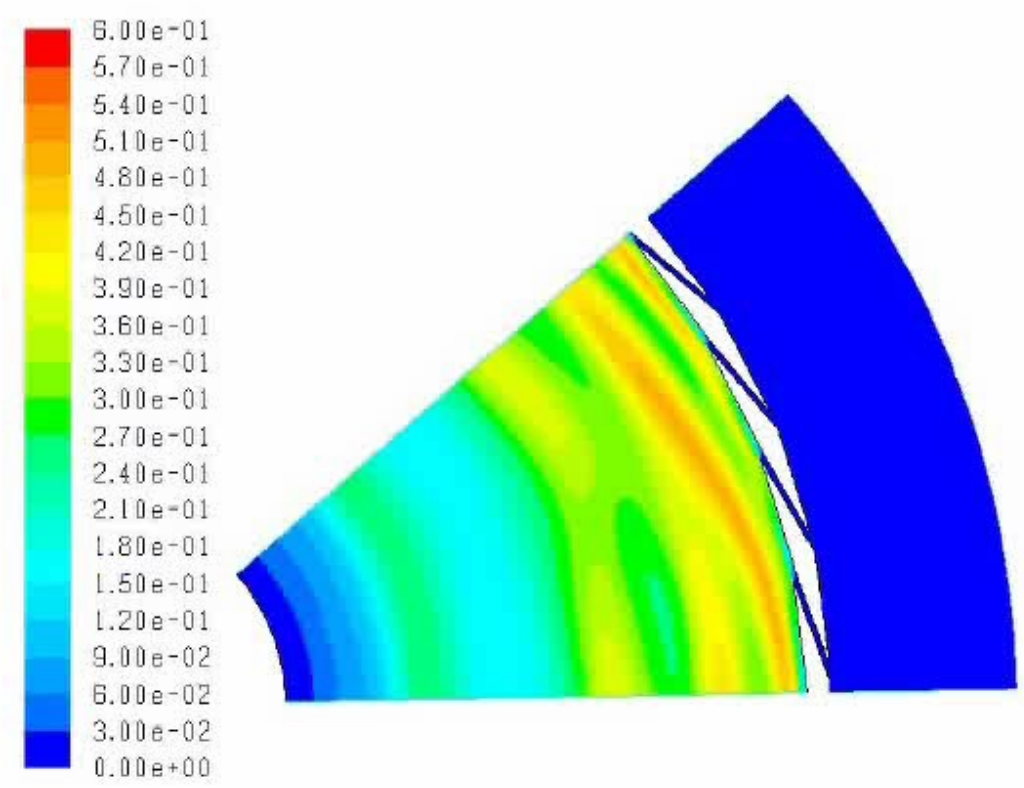




\section{Summary}

GSVRs have the potential to intensify processes

- High intrinsic mass/heat transfer can yield improved overall rates

- High solid volume fractions can reduce equipment size

\section{Biomass Pyrolysis Example}

- Stratification of solid phases to retain sand \& unreacted biomass

- Comparison to a static fluidize bed

- Comparable degree of char formation

- Increased tar and reduced pyrolysis gas formation

- Significantly opportunity for intensification in GSVR

- $3 x-5 x$ larger heat and mass transfer coefficients

- Ability to increase feed rates without biomass loss, but more $\Delta P$

\section{Future Projects}

- Direct measurement of solid velocities using PIV

- Experimental GSVR to examine heat transfer and reacting flows 


\section{Acknowledgements}

Lab. for Chemical Technology

- Prof. Guy Marin

- Prof. Geraldine Heynderickx

- Prof. Kevin van Geem

- Jelena Kovacevic

- dr. Maria Pantzali

- dr. Maarten Sabbe

- Georges Verenghen

Ghent University Resources

- High-Performance Computing Cluster

Funding

- Methusalem grant - Flemish government

- ERC Advanced Grant - MADPII
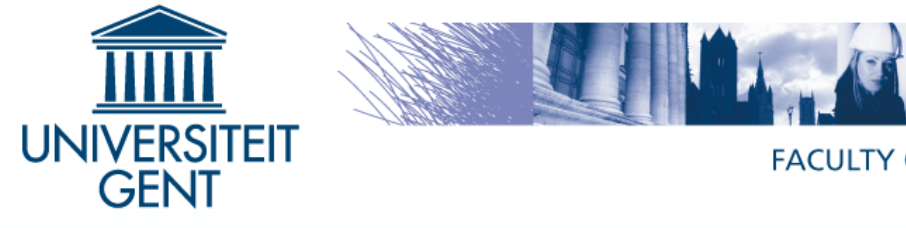

FACULTY OF ENGINEERING AND

ARCHITECTURE

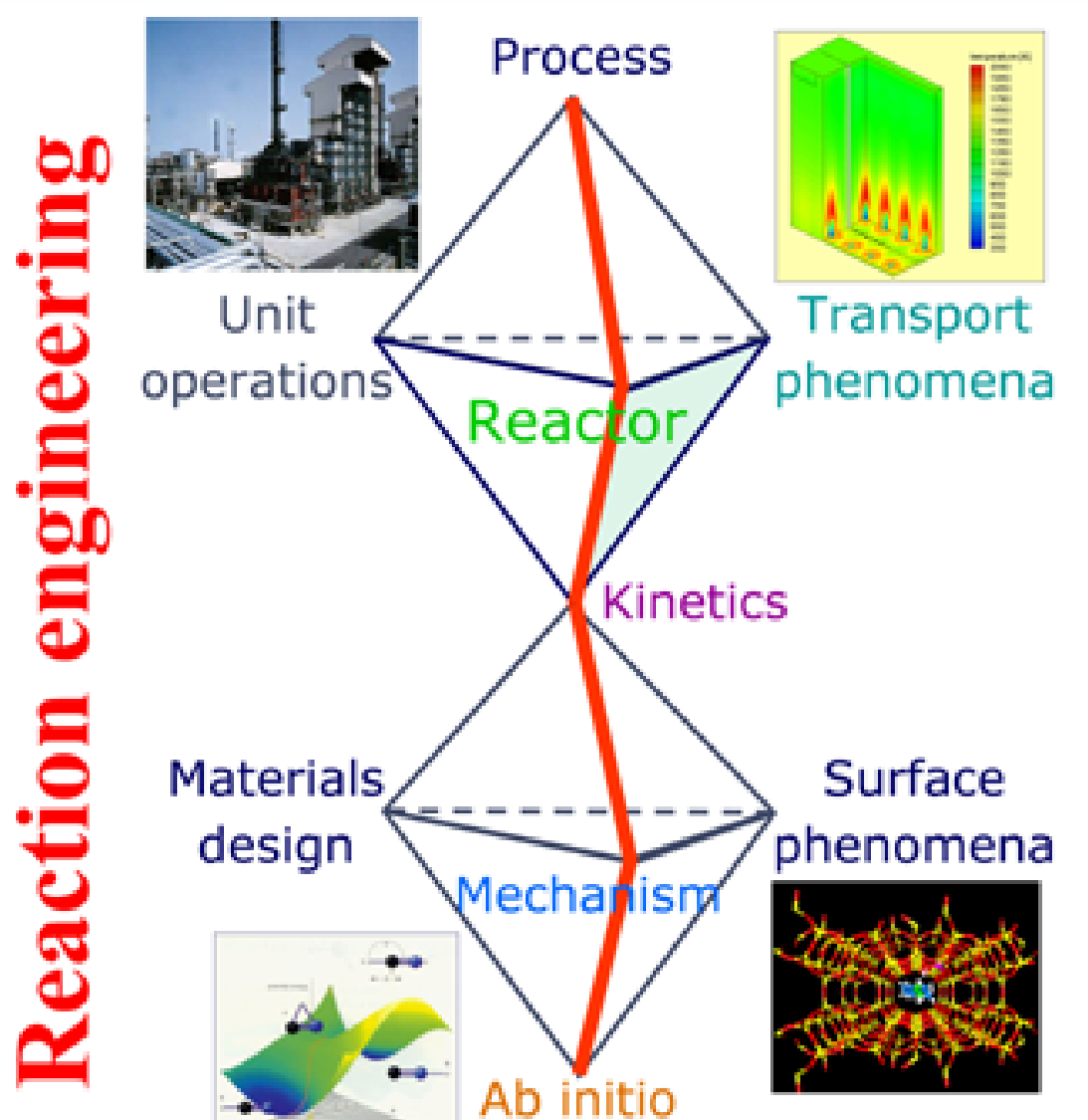




\section{Backup Slides}




\section{Flow Paths and Heat Transfer}

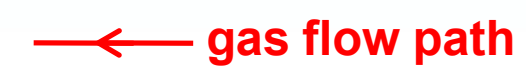

solid velocity

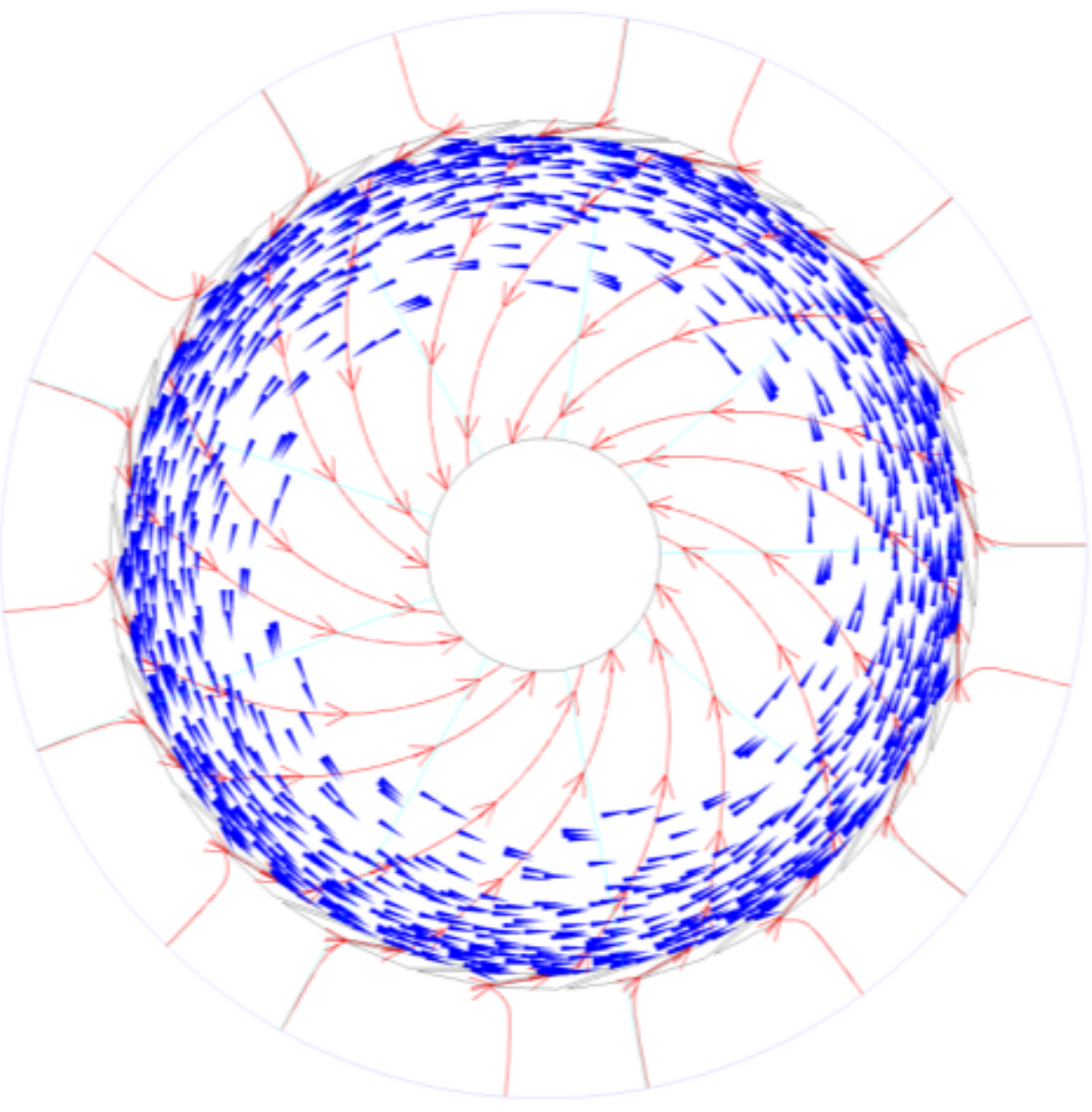

(a)
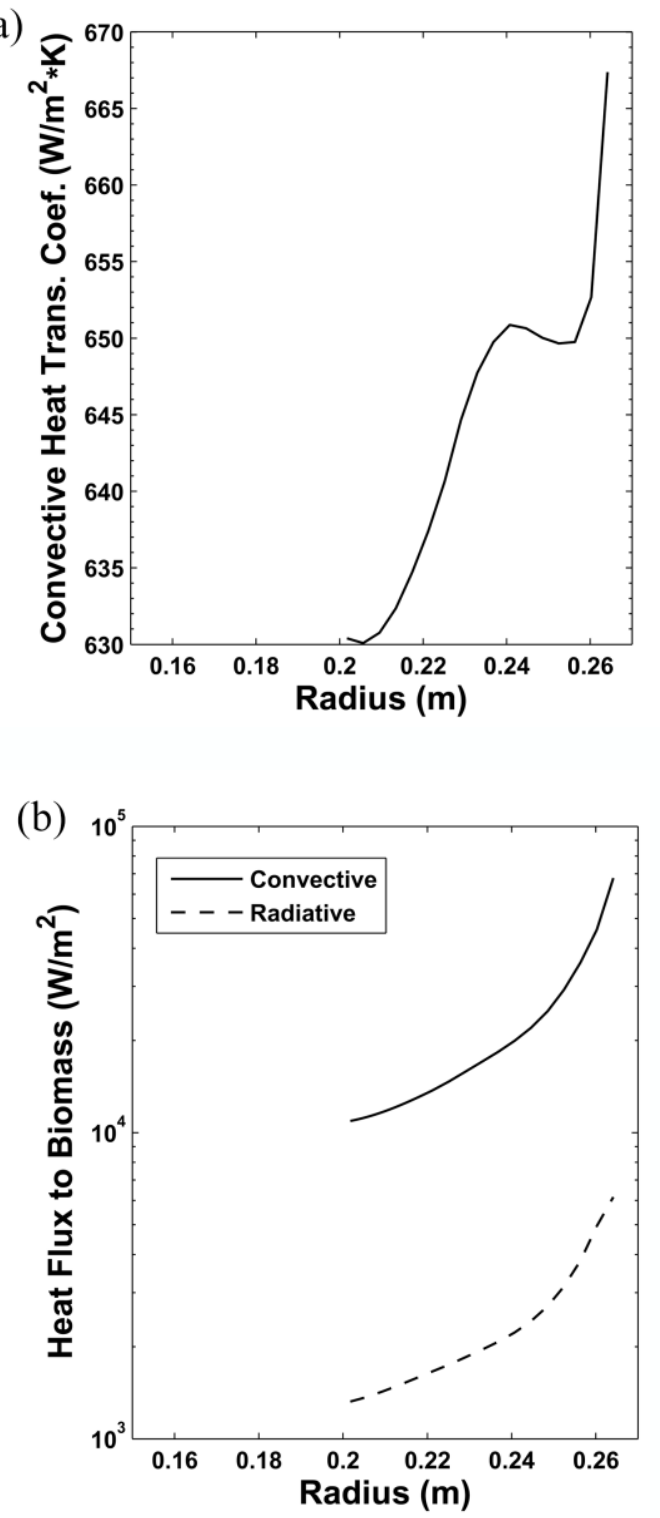


\section{Biomass Kinetic Model}

Table 1

Pyrolysis reaction mechanism and related data [9,47-49].

\begin{tabular}{|c|c|c|c|c|c|}
\hline & Reaction $^{\mathrm{a}, \mathrm{b}}$ & $\Delta H_{r x n}(\mathrm{~kJ} / \mathrm{kg})$ & $A_{f}(1 / s)$ & $E_{A}(\mathrm{~kJ} / \mathrm{mol})$ & $k_{\text {rxn }} @ 773 \mathrm{~K}(1 / \mathrm{s})$ \\
\hline $1 \mathrm{a}$ & $\mathrm{CL}_{v} \rightarrow \mathrm{CL}_{a}$ & 0 & $2.80 \times 10^{19}$ & 242.4 & 1170 \\
\hline 1b & $\mathrm{HC}_{v} \rightarrow \mathrm{HC}_{a}$ & 0 & $2.10 \times 10^{16}$ & 186.7 & 5080 \\
\hline $1 \mathrm{c}$ & $\mathrm{LG}_{v} \rightarrow \mathrm{LG}_{a}$ & 0 & $9.60 \times 108$ & 107.6 & 51.4 \\
\hline $2 \mathrm{a}$ & $\mathrm{CL}_{a} \rightarrow \mathrm{Tar}$ & 255 & $3.28 \times 10^{14}$ & 196.5 & 17.3 \\
\hline $2 b$ & $\mathrm{HC}_{a} \rightarrow \mathrm{Tar}$ & 255 & $8.75 \times 10^{15}$ & 202.4 & 184 \\
\hline $2 \mathrm{c}$ & $\mathrm{LG}_{a} \rightarrow \operatorname{Tar}$ & 255 & $1.50 \times 10^{9}$ & 143.8 & 0.287 \\
\hline $3 a$ & $\mathrm{CL}_{a} \rightarrow 0.35 \mathrm{Char}_{c}+2.6$ Pgas & -20 & $1.30 \times 10^{10}$ & 150.5 & 0.878 \\
\hline $3 b$ & $\mathrm{HC}_{a} \rightarrow 0.6 \mathrm{Char}_{h}+1.6$ Pgas & -20 & $2.60 \times 10^{11}$ & 145.7 & 37.1 \\
\hline $3 c$ & $\mathrm{LG}_{a} \rightarrow 0.75 \mathrm{Char}_{l}+$ Pgas & -20 & $7.70 \times 106$ & 111.4 & 0.228 \\
\hline 4 & Tar $\rightarrow$ 4Pgas & -42 & $4.25 \times 106$ & 108.0 & 0.214 \\
\hline
\end{tabular}

${ }^{a}$ Fractional char formation for CL, HC, and LG are $0.35,0.60$, and $0.75 \mathrm{~kg} \mathrm{char} / \mathrm{kg}$, respectively.

b Subscripts " $\mathrm{v}$ " and "a" indicate the virgin and activated forms of biomass, respectively.

Table 2

Pyrolysis species and relevant thermochemical and physical properties.

\begin{tabular}{|c|c|c|c|c|c|c|c|}
\hline & Component & Phase & $\mathrm{MW}^{\mathrm{a}}(\mathrm{g} / \mathrm{mol})$ & $\Delta H_{f}^{0}(\mathrm{~kJ} / \mathrm{mol})$ & $\rho_{s}\left(\right.$ dry $\left.\mathrm{kg} / \mathrm{m}^{3}\right)$ & $C_{p}(\mathrm{~J} /(\mathrm{kg} \mathrm{K}))$ & $\lambda(\mathrm{W} /(\mathrm{m} \mathrm{K}))$ \\
\hline 1 & $\mathrm{CL}_{a}$ & Biomass & 100 & 0 & 500 & 1400 & 0.209 \\
\hline 2 & $\mathrm{HC}_{v}$ & Biomass & 100 & 0 & 500 & 1400 & 0.209 \\
\hline 3 & $\mathrm{LG}_{v}$ & Biomass & 100 & 0 & 500 & 1400 & 0.209 \\
\hline 4 & $\mathrm{CL}_{a}$ & Biomass & 100 & 0 & 500 & 1400 & 0.209 \\
\hline 5 & $\mathrm{HC}_{a}$ & Biomass & 100 & 0 & 500 & 1400 & 0.209 \\
\hline 6 & $\mathrm{LG}_{a}$ & Biomass & 100 & 0 & 500 & 1400 & 0.209 \\
\hline 7 & Char $_{c}$ & Char & 100 & -45.27 & 175 & 1100 & 0.071 \\
\hline 8 & $\mathrm{Char}_{h}$ & Char & 100 & -17.53 & 300 & 1100 & 0.071 \\
\hline 9 & $\mathrm{Char}_{l}$ & Char & 100 & -9.77 & 375 & 1100 & 0.071 \\
\hline 10 & Tar & Gas & 100 & 25.5 & - & Phenol & Phenol \\
\hline 11 & Pgas & Gas & 25 & 5.33 & - & Ethylene & Ethylene \\
\hline 12 & $\mathrm{H}_{2} \mathrm{O}$ & Gas & 18.02 & - & - & - & - \\
\hline 13 & $\mathrm{~N}_{2}$ & Gas & 28.02 & - & - & - & - \\
\hline 14 & Sand & Sand & - & - & 2650 & 800 & 0.75 \\
\hline
\end{tabular}

The emissivity of all particulate phases was assumed to be 0.75 .

${ }^{a}$ The molecular masses of the biomass, char, and gas-phase pseudo-components are arbitrary and only affect the as-written stoichiometry of the reactions. 


\section{GSVR Biomass Simulations}

\begin{tabular}{|l|c|c|c|}
\hline Case name & $\begin{array}{c}\text { Air Feed Rate } \\
\mathbf{( k g / s )}\end{array}$ & $\begin{array}{c}\text { Biomass Feed } \\
\text { Rate } \mathbf{( k g / s )}\end{array}$ & $\begin{array}{c}\text { Biomass } \\
\text { Water } \%\end{array}$ \\
\hline Base & 0.22 & 0.035 & 10 \\
\hline No- $\mathrm{H}_{2} \mathrm{O}$ (high-T) & 0.22 & 0.035 & 0 \\
\hline $1.5 x-f l o w$ & 0.33 & 0.052 & 10 \\
\hline 2 -flow & 0.44 & 0.070 & 10 \\
No-sand & 0.22 & 0.035 & 10 \\
\hline No-sand/No- $\mathrm{H}_{2} \mathrm{O}$ (high-T) & 0.22 & 0.035 & 0 \\
& & & \\
& $\rightarrow$ Inlet Gas Temperature $=923 \mathrm{~K}$ & \\
& $\rightarrow$ Biomass Feed Temperature $=300 \mathrm{~K}$ &
\end{tabular}




\section{No-Sand Cases Results}

(a)

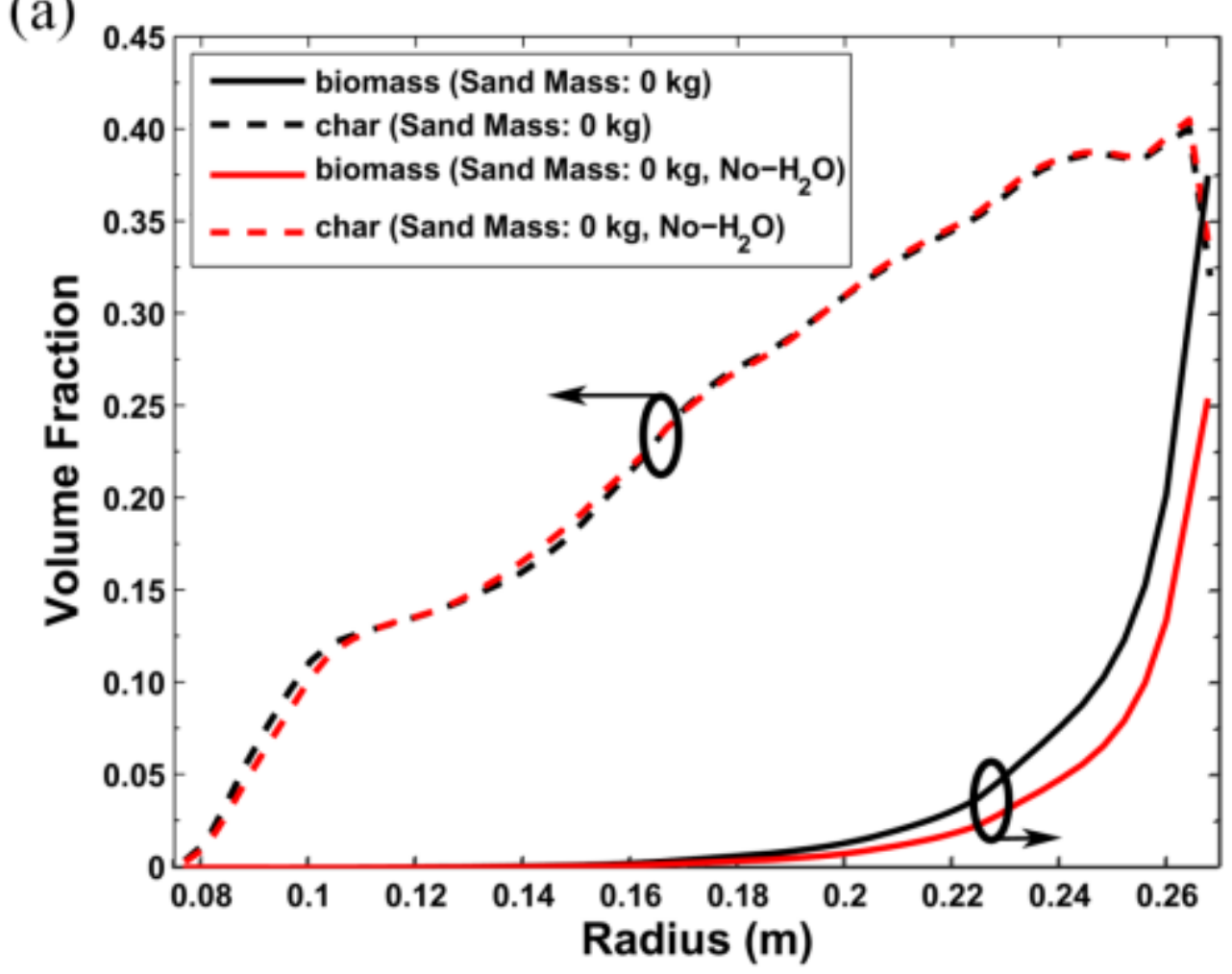

(b)

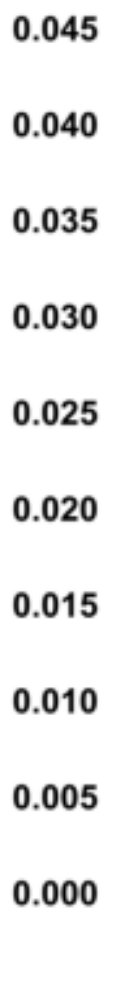

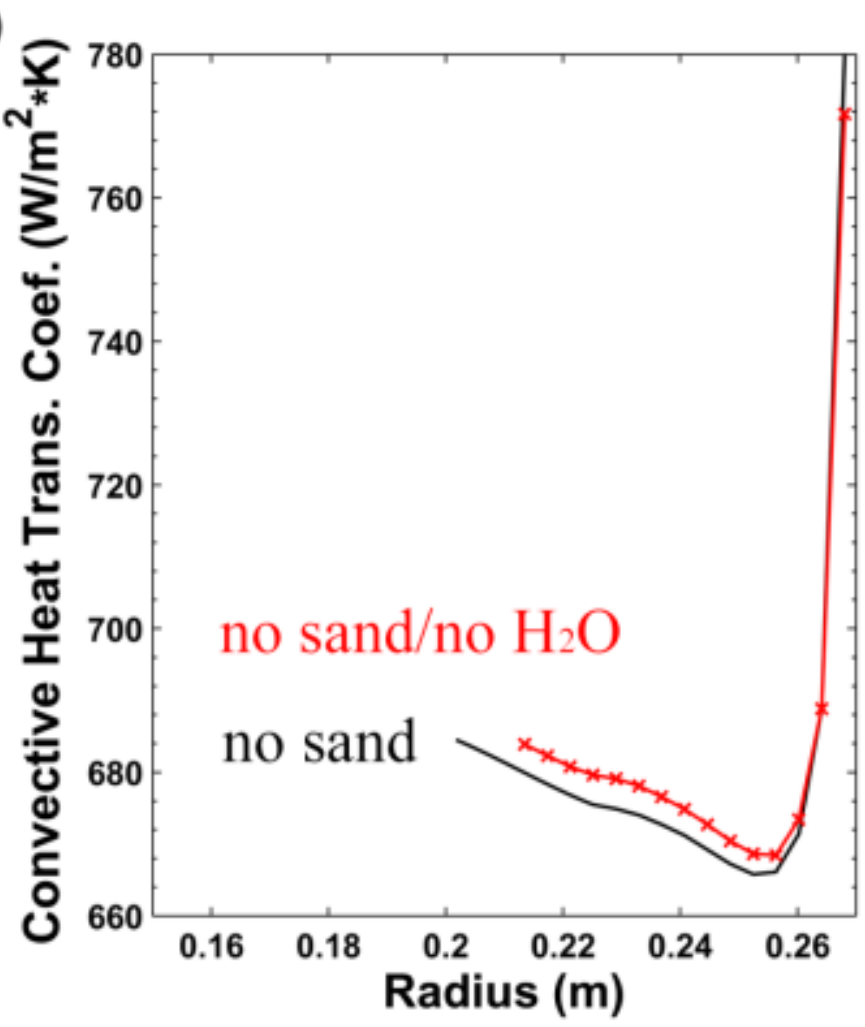

\begin{tabular}{lllllllll}
\hline Case name & $m_{\text {sand }}^{\text {ss }}(\mathrm{kg})$ & $m_{\text {bio }}^{\text {ss }}(\mathrm{kg})$ & $m_{\text {char }}^{\text {ss }}(\mathrm{kg})$ & $T_{\text {bio }}^{\text {avg }}(\mathrm{K})$ & $T_{\text {char }}^{\text {avg }}(\mathrm{K})$ & $T_{\text {sand }}^{\text {avg }}(\mathrm{K})$ & $T_{\text {gas }}^{\text {outlet }}(\mathrm{K})$ & $\dot{m}_{\text {char }}^{\text {out }}(\mathrm{kg} / \mathrm{s})$ \\
\hline Base & 5.0 & 0.056 & 0.119 & 721 & 753 & 761 & 751 & 0.0056 \\
No-sand & 0.0 & 0.056 & 1.939 & 727 & 751 & - & 750 & 0.0060 \\
$\mathrm{No}_{\mathrm{H}} \mathrm{O}$ & 5.0 & 0.036 & 0.123 & 734 & 787 & 794 & 784 & 0.0050 \\
No sand/No- $\mathrm{H}_{2} \mathrm{O}$ & 0.0 & 0.036 & 1.938 & 741 & 784 & - & 783 & 0.0056 \\
\hline
\end{tabular}




\section{Biomass Pyrolysis Product Distribution}

\begin{tabular}{lllll}
\hline Case name & Char (\%) & Tar (\%) & Pyrolysis gas (\%) \\
\hline Base & 16.1 & $(16.7 \%)^{\mathrm{a}}$ & 73.8 & 9.5 \\
$973 \mathrm{~K}$ & 14.6 & $(15.5 \%)^{\mathrm{a}}$ & 75.6 & 8.9 \\
$\mathrm{No}_{2} \mathrm{H} \mathrm{O}$ & 14.5 & $(15.1 \%)^{\mathrm{a}}$ & 76.0 & 8.9 \\
$1.5 \mathrm{x}-\mathrm{flow}$ & 16.3 & $(17.0 \%)^{\mathrm{a}}$ & 73.8 & 9.2 \\
$2 \mathrm{x}-\mathrm{flow}$ & 16.8 & $(17.6 \%)^{\mathrm{a}}$ & 73.3 & 9.1 \\
No-sand & 17.1 & $(17.1 \%)^{\mathrm{a}}$ & 73.6 & 9.3 \\
No sand/No- $\mathrm{H}_{2} \mathrm{O}$ & 16.0 & $(15.7 \%)^{\mathrm{a}}$ & 75.6 & 8.7 \\
\hline
\end{tabular}

${ }^{\text {a }}$ Char production fraction necessary to achieve a perfect mass balance over the reactor; mass balance errors ranged from $-0.9 \%$ to $+0.3 \%$. 


\section{"Full" 2D Simulations - Effect of Gravity}

- $\quad 0.4 \mathrm{~m}^{3} / \mathrm{s}$ air flow @ $1.225 \mathrm{~kg} / \mathrm{m}^{3}$ and $4.375 \mathrm{~kg}$ bed mass

- Run without gravity for 10 seconds

- Run 10 more sec. with/without gravity

- Quadrant view of solids VF:
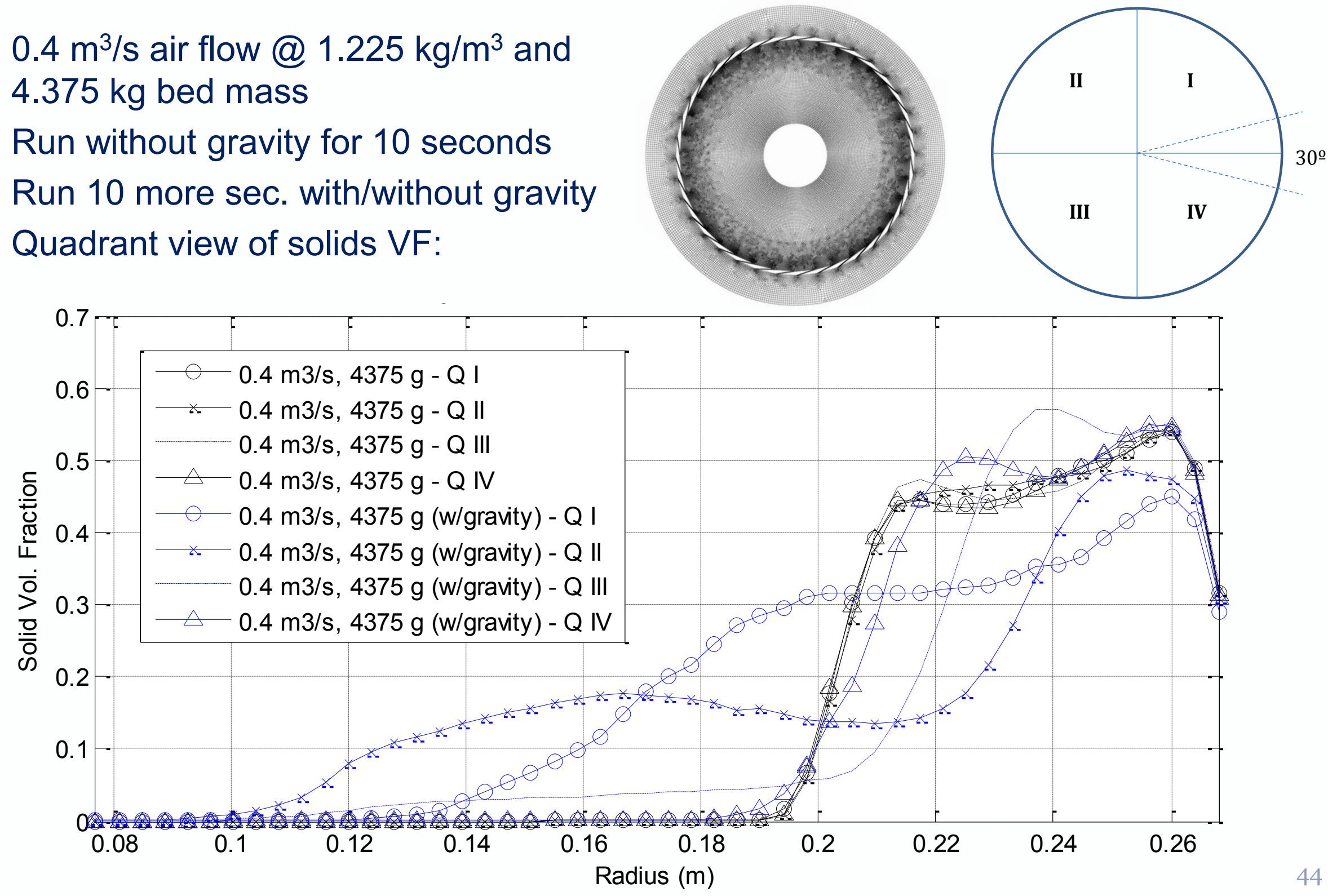


\section{"Full" 2D Simulations - Effect of Gravity}

- $\quad 0.8 \mathrm{~m}^{3} / \mathrm{s}$ air flow @ $1.225 \mathrm{~kg} / \mathrm{m}^{3}$ and $4.375 \mathrm{~kg}$ bed mass

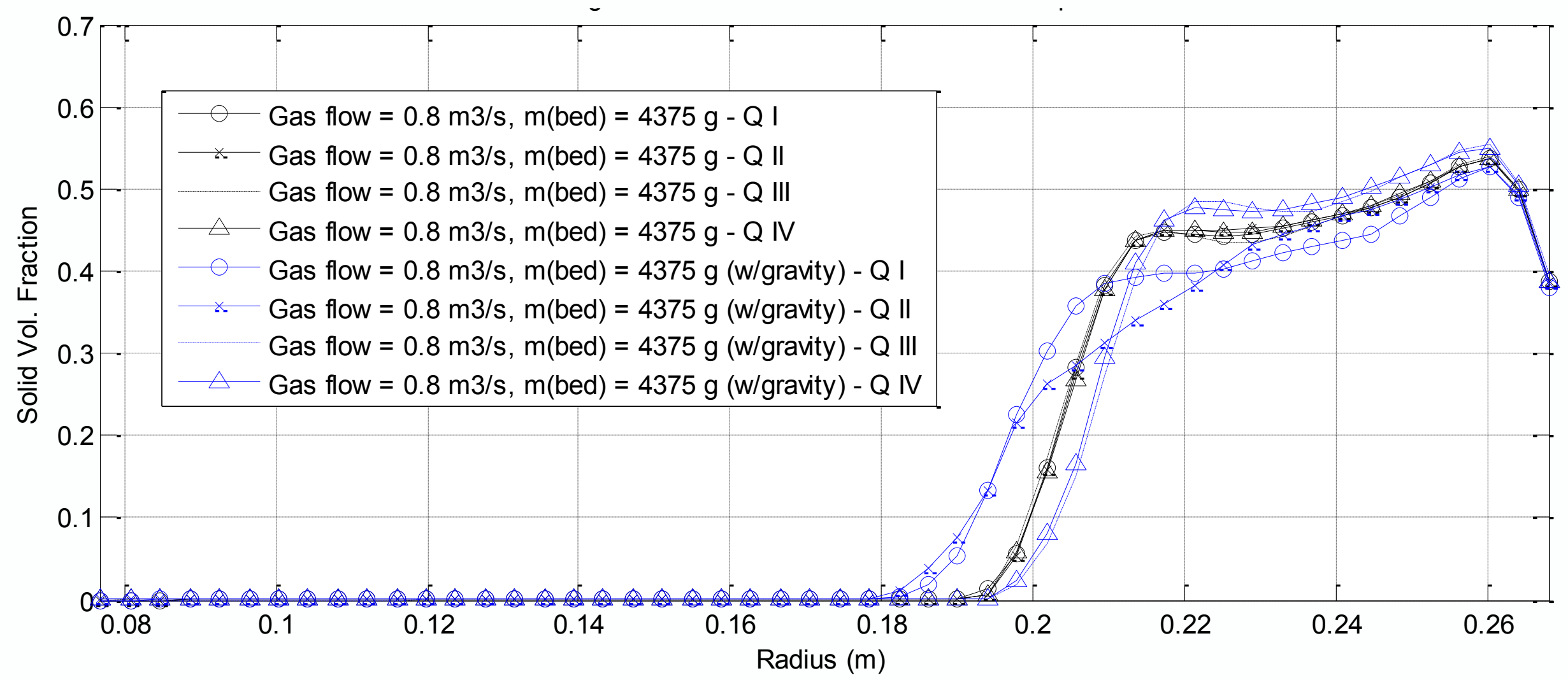



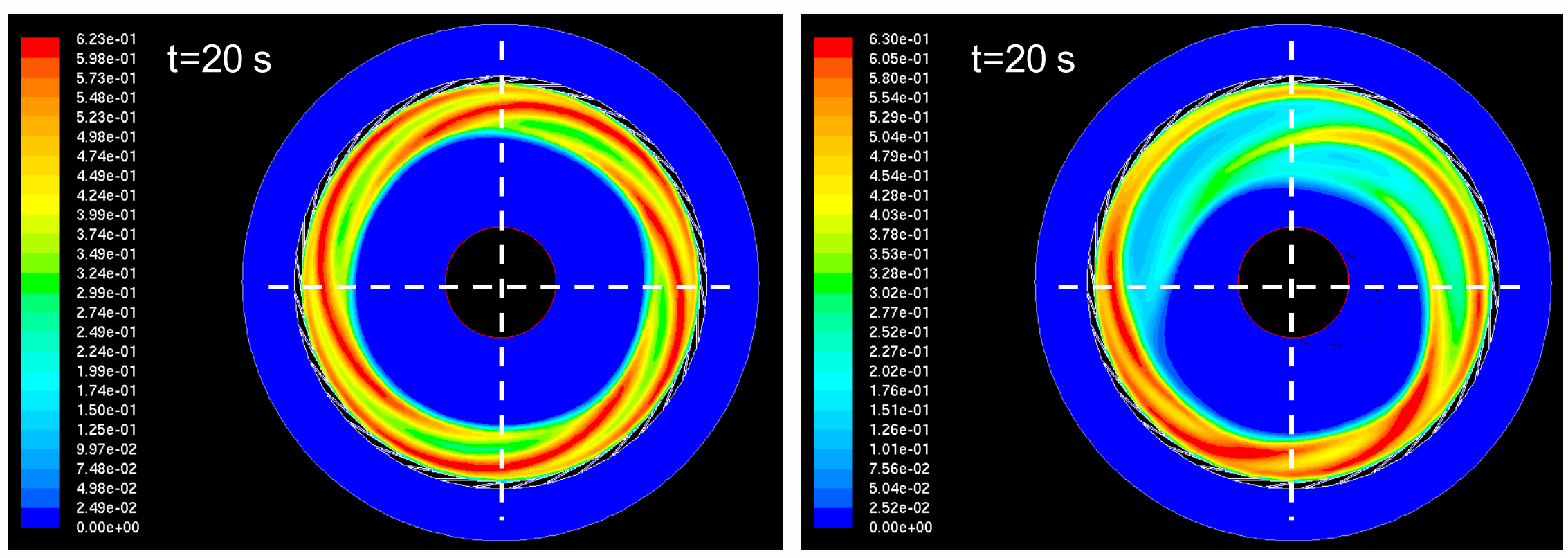

$0.98 \mathrm{~kg} / \mathrm{s}$ air, no gravity

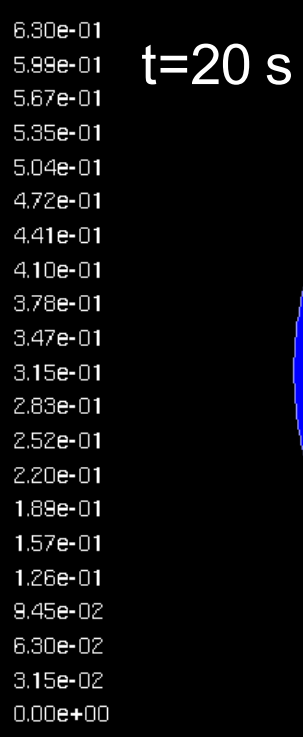

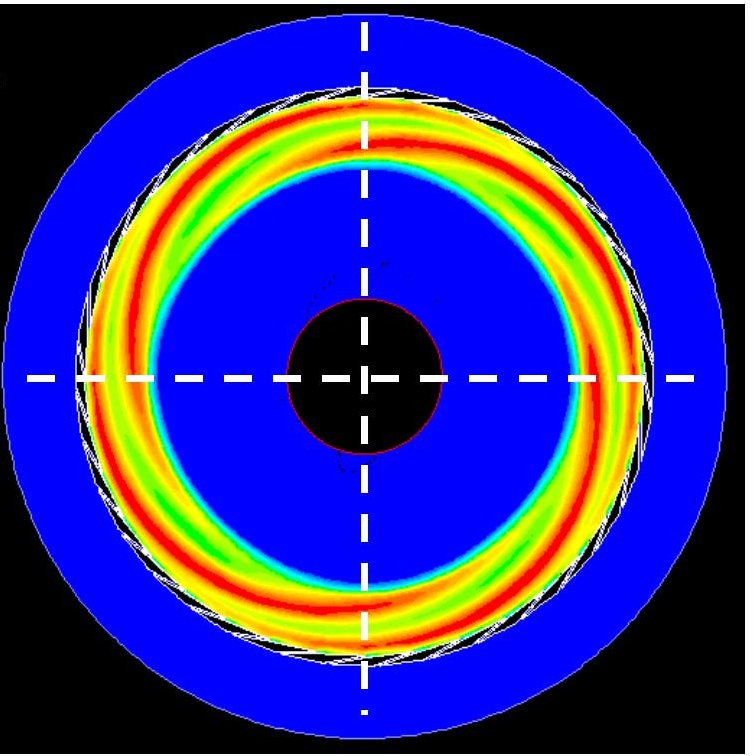

$0.98 \mathrm{~kg} / \mathrm{s}$ air, with gravity

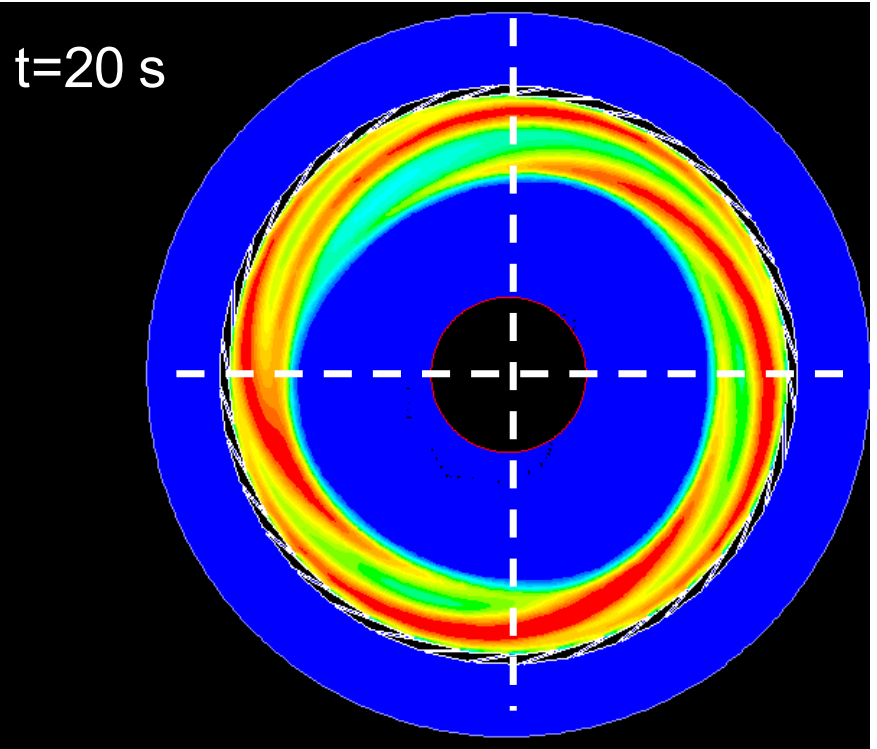




\section{On-going Model Refinement - PIV}

\section{Particle Image Velocimetry (PIV)}

- Allows for 2D particle velocity field near end-wall

- Final "major" observable for bulk validation

Raw image collection

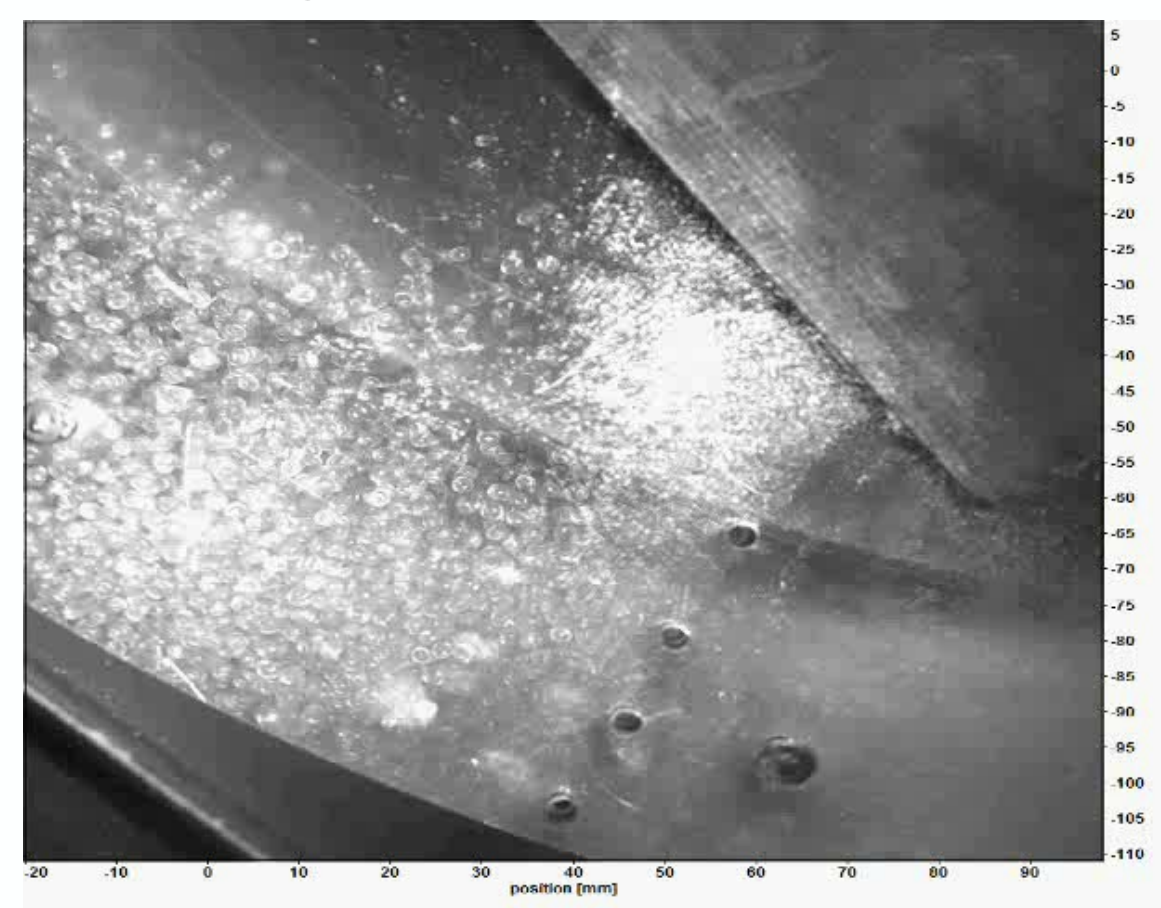

Processed velocity field

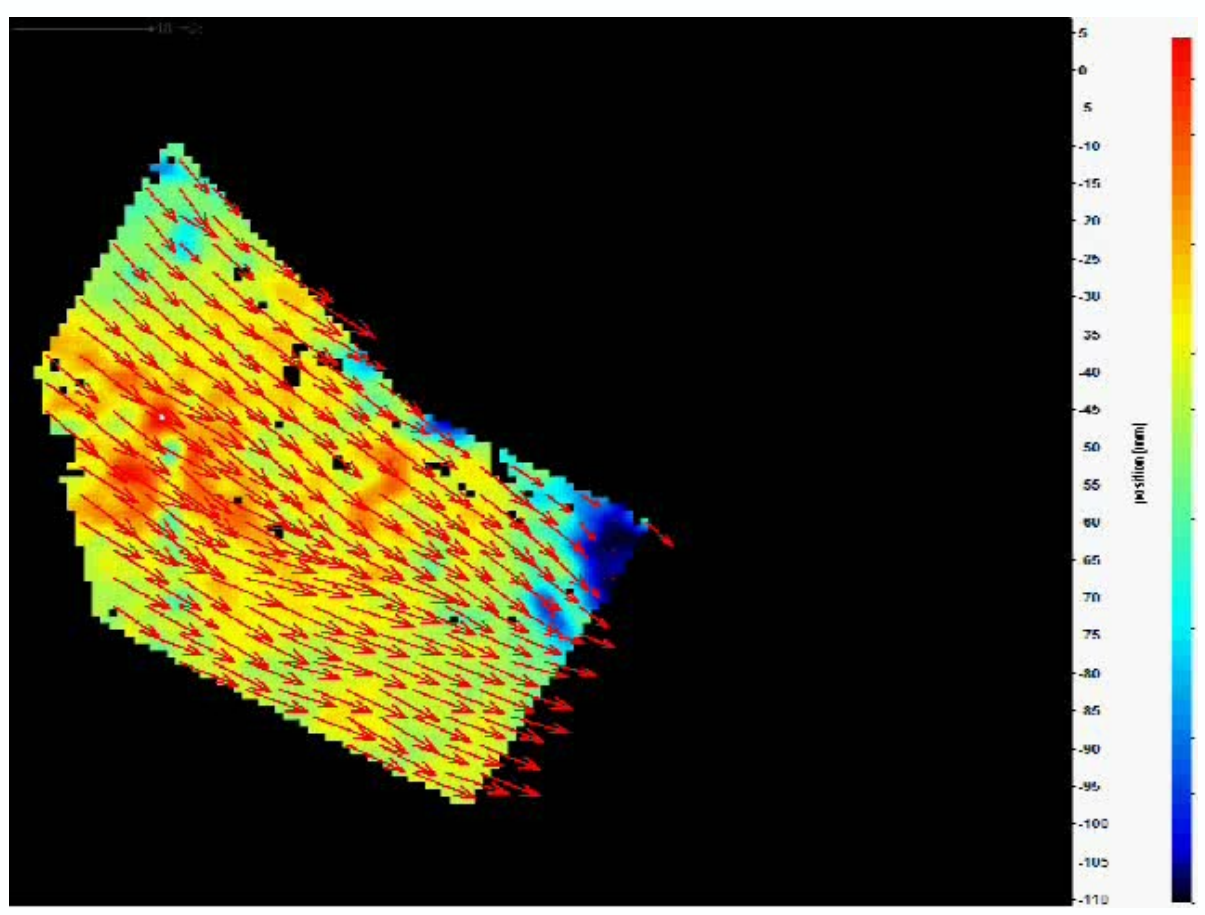




\section{2x-Flow VF Animation (back-up slide)}

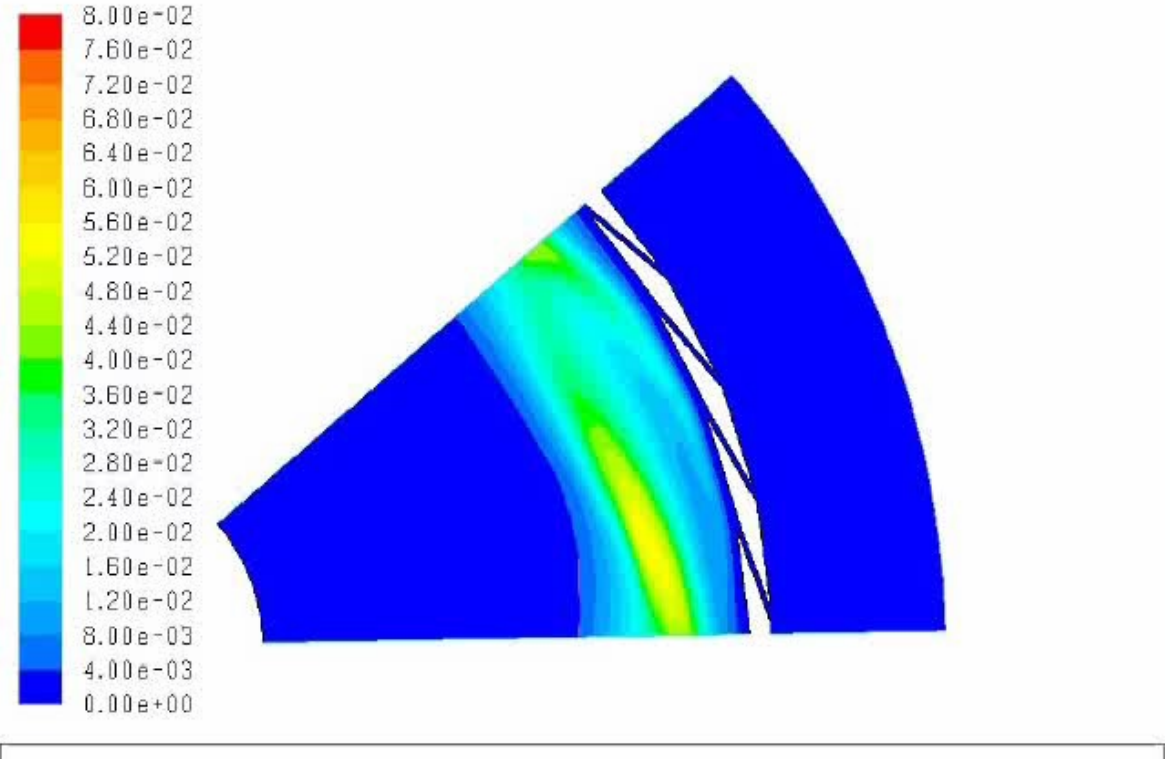
Contours of Volume fraction (biomass) (Time=1
ANSYS FLUENT 13.0 (2d, de. F

\section{Biomass \\ $\nearrow_{\text {Biomass }}$}

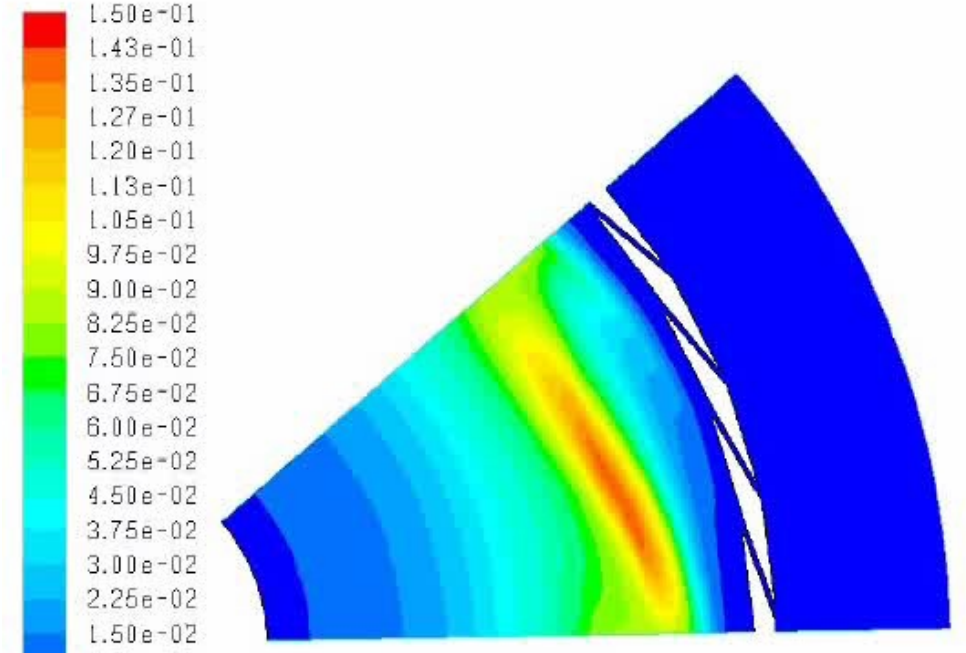

$4.50 \mathrm{e}-02$

$7.50 e-03$

$0.00 e+00$
$6.30 e-01$ $5.99 \mathrm{e}-01$ $5.67 e-01$ $5.35 e-01$ $5.04 \mathrm{e}-01$ $4.72 \mathrm{e}-01$ $4.41 e-01$ $4.10 e-01$ $3.78 \mathrm{e}-01$ $3.47 e-01$ $3.15 \mathrm{e}-01$ $2.83 e-01$ $2.52 \mathrm{e}-01$

$2.20 e-01$ $2.20 e-01$
$1.89 e-01$ $1.57 \mathrm{e}-01$ $1.26 e-01$ $9.45 \mathrm{e}-02$ $6.30 e-02$ $3.15 e-02$ $0.00 e+00$

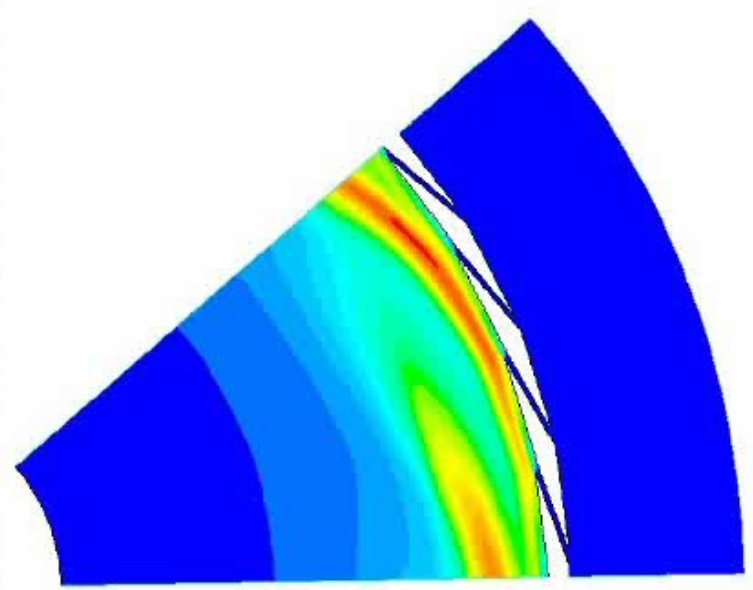

Time $=1.00000+021$ Apr 21,2012 [2d, dp, pbns, eulerian, spe, rke, transient] হ

Char

\section{Total solids} (biomass, char, sand) 

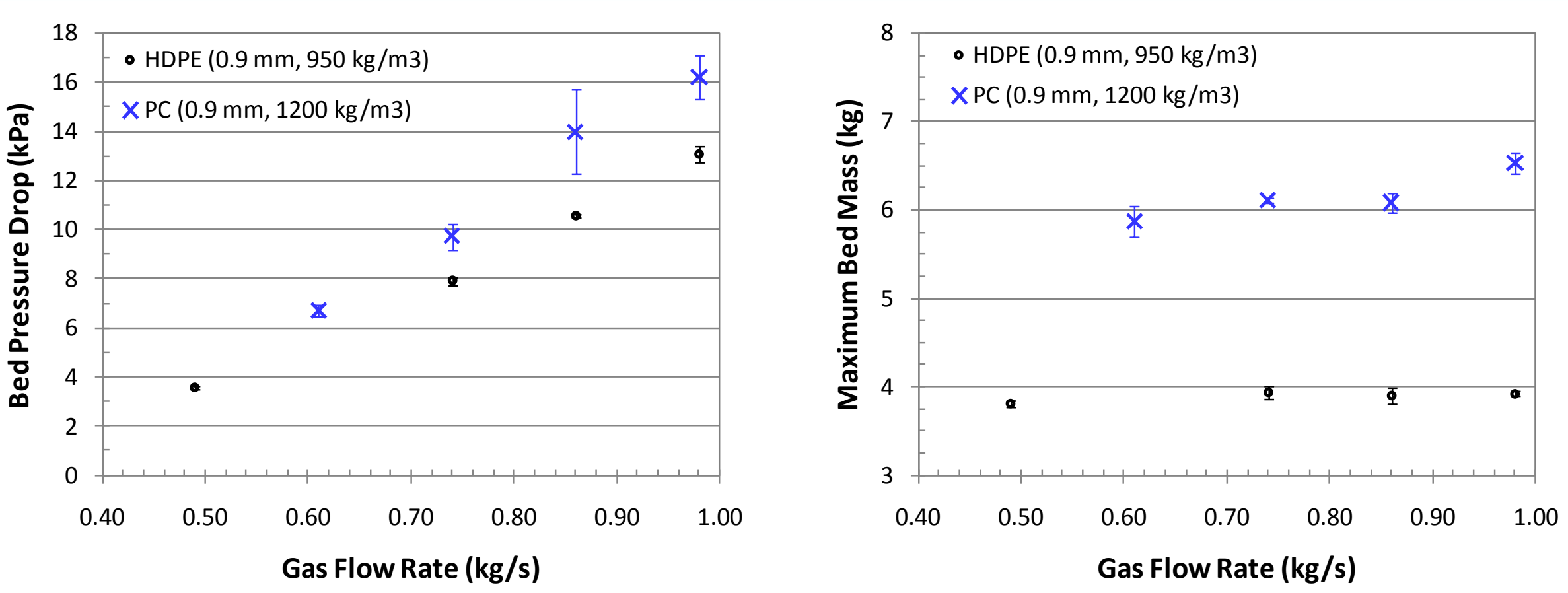

\section{Scaled Bed}

Pressure Drop

$$
\Delta \Pi=\frac{\Delta P_{\text {bed }}}{\rho_{s} g H_{\text {bed }} \varepsilon_{s}}
$$

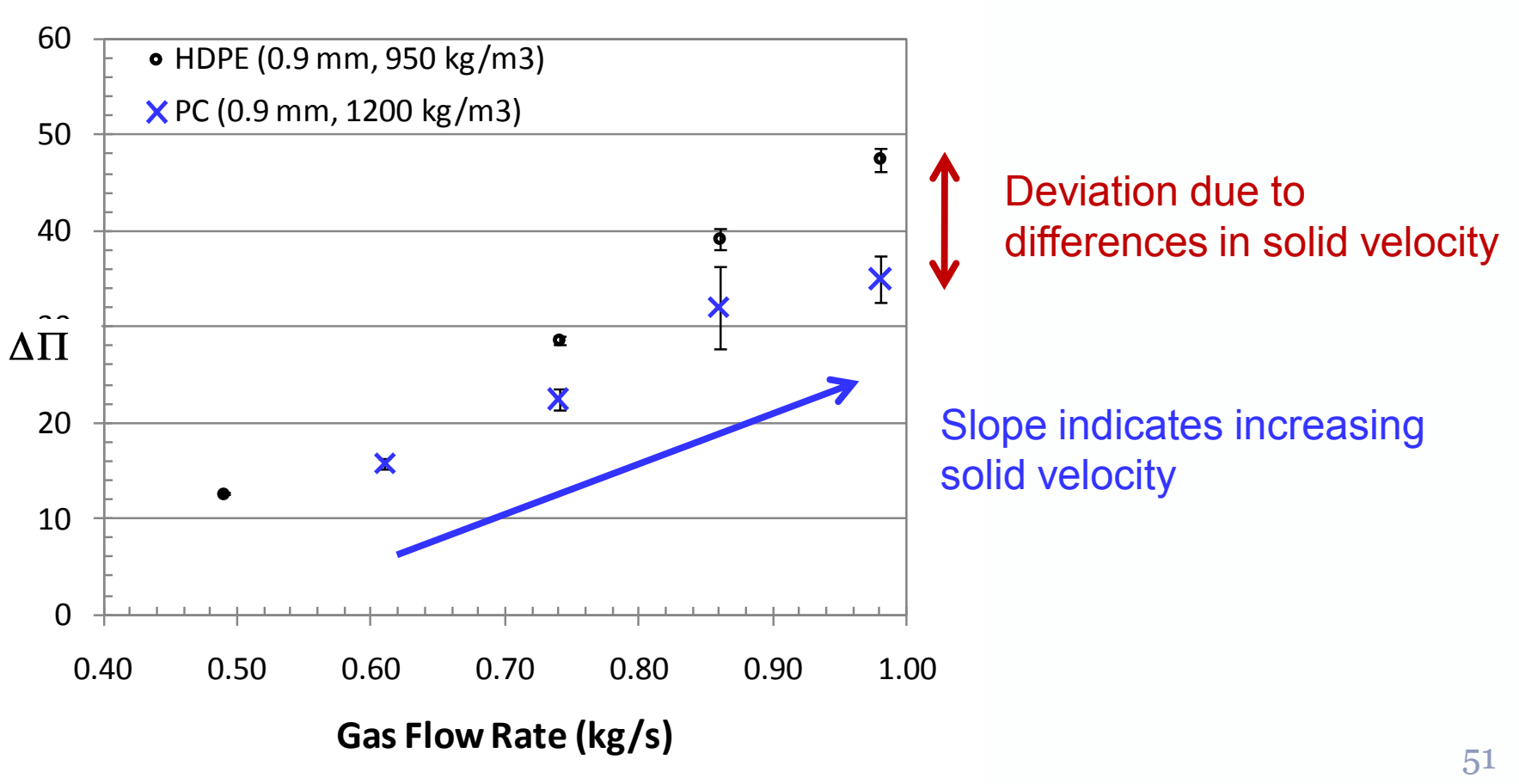




\section{CFD Example Movies}

$3 \mathrm{D}, 0.74 \mathrm{~kg} / \mathrm{s}$ air, $3250 \mathrm{~g}$ bed

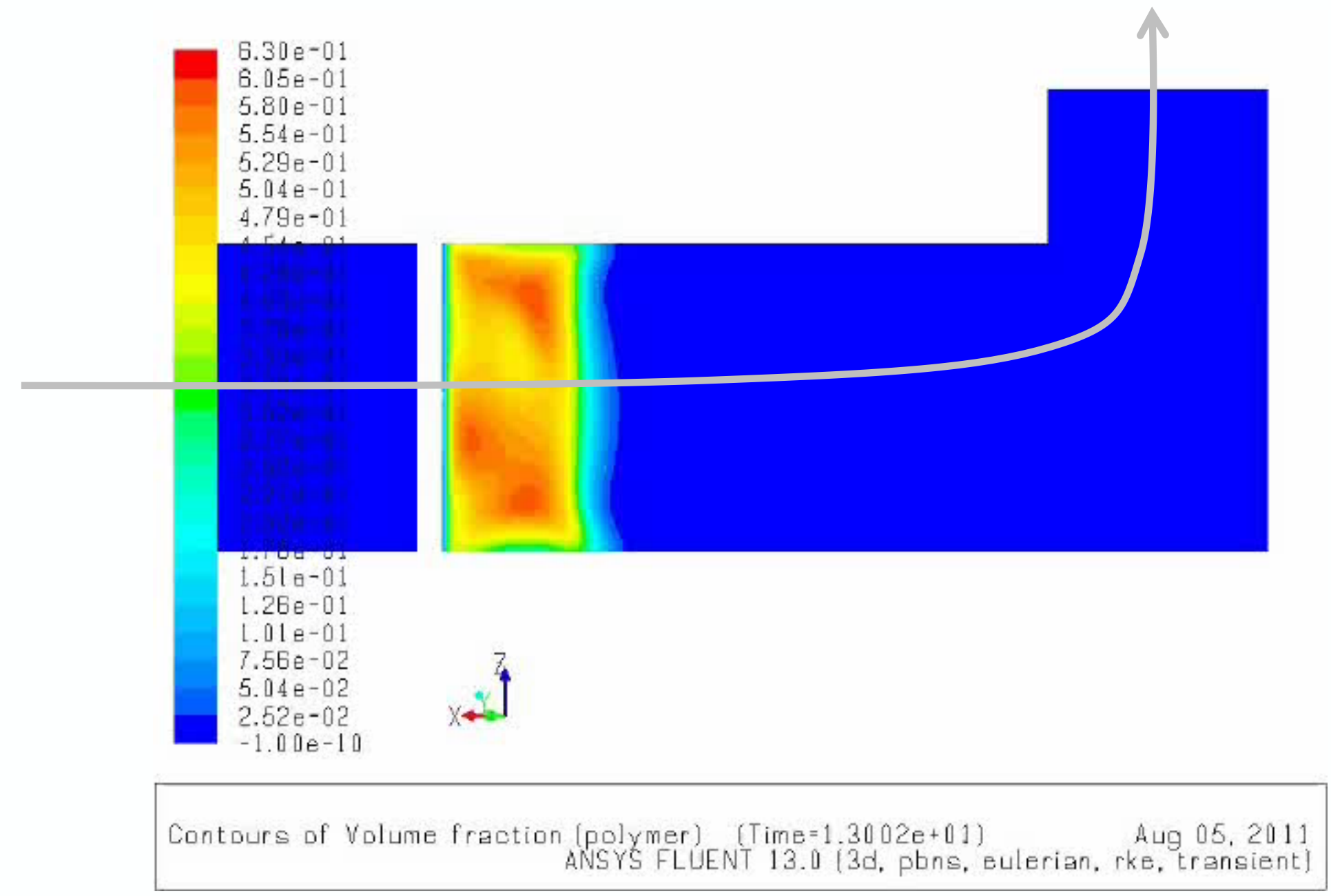

